\title{
Higgs mass predictions of public NMSSM spectrum generators
}

\author{
Florian Staub ${ }^{\mathrm{a}, *}$, Peter Athron ${ }^{\mathrm{b}}$, Ulrich Ellwanger ${ }^{\mathrm{c}}$, Ramona Gröber ${ }^{\mathrm{d}}$, Margarete Mühlleitner $^{\mathrm{e}}$, \\ Pietro Slavich ${ }^{\mathrm{f}}$, Alexander Voigt ${ }^{\mathrm{g}}$ \\ ${ }^{a}$ Theory Division, CERN, 1211 Geneva 23, Switzerland \\ ${ }^{b}$ ARC Centre of Excellence for Particle Physics at the Terascale, School of Physics, Monash University, Melbourne \\ VIC 3800, Australia \\ ${ }^{c}$ LPT, UMR 8627, CNRS, Université de Paris-Sud, 91405 Orsay, France, and School of Physics and Astronomy, \\ University of Southampton, Highfield, Southampton SO17 1BJ, UK \\ ${ }^{d}$ Sezione di Roma Tre, Via della Vasca Navale 84, I-00146 Roma, Italy \\ ${ }^{e}$ Institute for Theoretical Physics, Karlsruhe Institute of Technology, Wolfgang-Gaede-Str. 1, 76131 Karlsruhe, \\ Germany \\ ${ }^{f}$ LPTHE, UPMC Univ. Paris 06, Sorbonne Universités, UMR 7589, 4 Place Jussieu, F-75252, Paris, France \\ LPTHE, CNRS, UMR 7589, 4 Place Jussieu, F-75252, Paris, France \\ ${ }^{g}$ Deutsches Elektronen-Synchrotron DESY, Notkestraße 85, D-22607 Hamburg, Germany
}

\begin{abstract}
The publicly available spectrum generators for the NMSSM often lead to different predictions for the mass of the standard model-like Higgs boson, even though they all implement two-loop computations in the $\overline{\mathrm{DR}}$ renormalization scheme. Depending on the parameter point, the differences can exceed $5 \mathrm{GeV}$, and even reach $8 \mathrm{GeV}$ for moderate superparticle masses of up to $2 \mathrm{TeV}$. It is shown here that these differences can be traced back to the calculation of the running standard model parameters entering all calculations, to the approximations used in the two-loop corrections included in the different codes, and to different choices for the renormalization conditions and scales. In particular, the importance of the calculation of the top Yukawa coupling is pointed out.
\end{abstract}

\section{Introduction}

Now that run II of the Large Hadron Collider (LHC) has started, either the first clear hints for new physics beyond the standard model (BSM) will finally show up or many well-motivated theories for BSM physics will come under even greater pressure. While in run I a scalar was discovered [1, 2] which has all properties of the long-expected Higgs boson of the standard model (SM), at the same time stringent limits were placed on the simplest scenarios for new physics. In particular, the minimal realization of supersymmetry - the minimal supersymmetric standard model (MSSM) - has lost at least some of its appeal: no hints for supersymmetric (SUSY) particles were found, and the observed value of the SM-like Higgs mass requires either multi-TeV stop masses or special values of the stop-mixing parameter. However, if the stops are heavy and the MSSM is assumed to be a valid description of nature far above the electroweak scale, then a large fine-tuning is required to stabilize the vacuum expectation value (VEV) of the Higgs field. On the other hand, the large stop mixing needed to generate a Higgs mass of $125 \mathrm{GeV}$ for stop masses around one $\mathrm{TeV}$ turns out to be dangerous because of charge and color breaking minima [3, 4, 5, 6, 7]. This leads to the question of whether the MSSM is really a natural completion of the SM and has attracted more interest in other SUSY scenarios. This is especially the case for SUSY models that predict a larger Higgs mass already at tree level. The best-studied examples are models with singlet extensions, where the interaction between

\footnotetext{
${ }^{*}$ Corresponding author

URL: florian.staub@cern.ch (Florian Staub), peter.athron@coepp.org.au (Peter Athron), Ulrich.Ellwanger@th.u-psud.fr (Ulrich Ellwanger), groeber@roma3.infn.it (Ramona Gröber), maggie@particle.uni-karlsruhe.de (Margarete Mühlleitner), slavich@lpthe.jussieu.fr (Pietro Slavich), alexander.voigt@desy.de (Alexander Voigt) 
the Higgs fields and the gauge singlet can raise the Higgs mass [8, 9, 10] and significantly reduce the fine-tuning [11, 12, 13, 14, 15, 16, 17, 18, 19, 20]. A $\mathbb{Z}_{3}$ symmetry is often introduced to forbid all dimensionful parameters in the superpotential and to solve the $\mu$ problem of the MSSM at the same time [9]. This realization of a singlet extension of the MSSM is the next-to-minimal supersymmetric standard model (NMSSM). The NMSSM comes with a rich collider phenomenology [21, 22, 23], and also provides possibilities to hide SUSY at the LHC [24].

To confront the NMSSM with the measured Higgs mass, a precise calculation is necessary. The first calculations of radiative corrections to the Higgs masses in the NMSSM assumed an effective field theory below the SUSY scale (the scale of the stop/sbottom masses), and integrated the renormalization group equations (RGEs) for the corresponding couplings from the SUSY scale to the weak scale [25, 26, 27]. One-loop correction ${ }^{\mathbb{1}}$ of $\mathcal{O}\left(\alpha_{t}+\alpha_{b}\right)$ in the effective potential approximation have been computed in Refs. [28, 29, 30, 31. Two-loop corrections of $\mathcal{O}\left(\alpha_{s} \alpha_{t}+\alpha_{t}^{2}\right)$ have been included in the NMSSM in the leading-logarithmic approximation (LLA) in Refs. 32, 33, and one-loop corrections to the SM-like Higgs mass including NMSSM-specific and electroweak couplings in the LLA in Ref. [34. Full oneloop calculations including the momentum dependence have been performed in the $\overline{\mathrm{DR}}$ renormalization schem ${ }^{2}$ [36, 37, or in a mixed on-shell (OS)-DR scheme [38, 39]; at the two-loop level, the dominant $\mathcal{O}\left(\alpha_{s}\left(\alpha_{b}+\alpha_{t}\right)\right)$ corrections in the $\overline{\mathrm{DR}}$ scheme have become available a few years ago [36]. The two-loop corrections involving only superpotential couplings such as Yukawa and singlet interactions [40, and a two-loop calculation of the $\mathcal{O}\left(\alpha_{s} \alpha_{t}\right)$ corrections with the top/stop sector renormalized either in the OS scheme or in the $\overline{\mathrm{DR}}$ scheme [41], have also been provided recently.

At the moment, five public spectrum generators for the NMSSM are available which make use of these results to compute the Higgs-boson masses and mixing matrices in the NMSSM for a given parameter point: the stand-alone codes NMSSMTools [42, 43, 44], SOFTSUSY [45, 46, 47] and NMSSMCALC [48], as well as NMSSM versions of FlexibleSUSY [49] and SPheno [50, 51, which are based on SARAH [52, 53, 54, 55, 56]. The various tools, however, often make predictions that differ by several GeV for the mass of the SM-like Higgs boson even if using the same renormalization scheme. These differences are bigger than the ones observed between codes that calculate the Higgs mass in the MSSM [57, 58]. The aim of this work is to identify the origin of these deviations for the $\overline{\mathrm{DR}}$ calculation, and to determine the numerical significance of each difference between the codes. A comparison between the $\overline{\mathrm{DR}}$ and OS calculations, which would provide an estimate of the theoretical uncertainty, is beyond the scope of this analysis and will be given elsewhere.

The paper is organized as follows. In sec. 2 we introduce the model and the conventions used. In sec. 3 the different codes and their features are presented, and the results obtained in six test points when using the codes out-of-the box are shown. The differences in the Higgs-mass calculation between these codes are discussed in sec. 4. Here, we concentrate on a SUSY scale input, while we address additional effects in a GUT scenario in sec. 5. We conclude in sec. 6.

\section{The NMSSM and its conventions}

We consider in the following a model with the particle content of the MSSM extended by a gauge singlet superfield $\hat{S}$. The general, renormalizable and $R$-parity conserving superpotential for this model in terms of the (hatted) doublet $\left(\hat{H}_{u}, \hat{H}_{d}\right)$ and singlet superfields reads

$$
\mathcal{W}_{S}=\mathcal{W}_{\text {Yukawa }}+\frac{1}{3} \kappa \hat{S}^{3}+(\mu+\lambda \hat{S}) \hat{H}_{u} \hat{H}_{d}+\frac{1}{2} \mu_{s} \hat{S}^{2}+t_{s} \hat{S}
$$

with

$$
\mathcal{W}_{\text {Yukawa }}=Y_{u} \hat{Q} \hat{U} \hat{H}_{u}+Y_{d} \hat{Q} \hat{D} \hat{H}_{d}+Y_{e} \hat{L} \hat{E} \hat{H}_{d}
$$

\footnotetext{
${ }^{1}$ We define $\alpha_{t, b, \tau}=Y_{t, b, \tau}^{2} /(4 \pi)$, where $Y_{t, b, \tau}$ are the third-family Yukawa couplings, and adopt analogous definitions for $\alpha_{\lambda}$ and $\alpha_{\kappa}$, where $\lambda$ and $\kappa$ are the NMSSM-specific couplings in eq. 11 below. We follow the standard convention of denoting, e.g., by $\mathcal{O}\left(\alpha_{t}\right)$ the one-loop corrections to the Higgs masses that are in fact proportional to $\alpha_{t} m_{t}^{2}$, i.e. proportional to $Y_{t}^{4}$. Similar abuses of notation affect the other one- and two-loop corrections.

${ }^{2}$ Strictly speaking, all calculations have been done in the $\overline{\mathrm{DR}}^{\prime}$-scheme where the $\epsilon$ scalars are decoupled [35].
} 
where we have suppressed color and isospin indices in every term. In general, the couplings in eq. (1) are complex parameters, and those in eq. (2) are complex $3 \times 3$ matrices. The most general soft SUSY-breaking terms for this theory are given by

$$
\begin{aligned}
V_{\text {soft }} & =m_{s}^{2}|S|^{2}+m_{H_{u}}^{2}\left|H_{u}\right|^{2}+m_{H_{d}}^{2}\left|H_{d}\right|^{2}+\tilde{f}^{\dagger} m_{\tilde{f}}^{2} \tilde{f} \\
& +M_{1} \lambda_{B}^{2}+M_{2} \lambda_{W}^{2}+M_{3} \lambda_{g}^{2}+T_{d} \tilde{q} \tilde{d} H_{d}+T_{u} \tilde{q} \tilde{u} H_{u}+T_{e} \tilde{e} \tilde{l} H_{d} \\
& +\left(B_{\mu} H_{u} H_{d}+\frac{1}{2} B_{s} S S+\chi_{s} S+T_{\lambda} S H_{u} H_{d}+\frac{1}{3} T_{\kappa} S^{3}+\text { h.c. }\right),
\end{aligned}
$$

with the left-handed squark and slepton doublets $\tilde{f}=\tilde{q}$ and $\tilde{l}$ and the right-handed squarks and sleptons $\tilde{f}=\tilde{u}_{R}, \tilde{d}_{R}, \tilde{e}_{R}$. All generation indices are suppressed. The soft SUSY-breaking masses $m_{s}^{2}$, $m_{H_{d}}^{2}$ and $m_{H_{u}}^{2}$ are always real, and the sfermion masses $m_{\tilde{f}}^{2}$ are hermitian $3 \times 3$ matrices, while all other soft terms can be complex. The soft SUSY-breaking trilinears $T_{\lambda}$ and $T_{\kappa}$ are often expressed in an alternative way, as the product of the corresponding superpotential coupling times a free parameter with the dimensions of a mass

$$
T_{\lambda} \equiv \lambda A_{\lambda}, \quad T_{\kappa} \equiv \kappa A_{\kappa} .
$$

Similarly, the trilinear soft SUSY-breaking interactions involving sfermions can be expressed as

$$
T_{i} \equiv Y_{i} A_{i}, \quad i=u, d, e .
$$

It is common to introduce a $\mathbb{Z}_{3}$ symmetry to forbid all dimensionful parameters (i.e., $\mu, \mu_{s}$ and $t_{s}$ ) in eq. (1). This solves the $\mu$ problem of the MSSM [59] - at the price of introducing a domain wall problem 60 - and leads to the NMSSM, where the surviving terms in the superpotential are

$$
\mathcal{W}_{\mathrm{NMSSM}}=\mathcal{W}_{\text {Yukawa }}+\frac{1}{3} \kappa \hat{S}^{3}+\lambda \hat{S} \hat{H}_{u} \hat{H}_{d}
$$

and the soft SUSY-breaking terms $B_{\mu}, B_{s}$ and $\chi_{s}$ in eq. (3) also vanish.

For suitable values of the remaining $s$-dependent SUSY-breaking terms $m_{s}$ and $T_{\kappa}$, a VEV $v_{s}$ for the scalar singlet $S$ of the order of the SUSY-breaking scale is generated. This dynamically generates the $\mu$ term which gives masses to the higgsinos,

$$
\mu_{\mathrm{eff}}=\lambda\langle S\rangle \text {. }
$$

Note, there are two common conventions: $\langle S\rangle=v_{s}$ or $\langle S\rangle=v_{s} / \sqrt{2}$. The SUSY Les Houches Accord (SLHA) 61, 62 does not fix a convention, and the codes compared here make different choices. However in this text we will use the latter convention. Finally, after electroweak symmetry breaking (EWSB) where also the neutral components of $H_{d}$ and $H_{u}$ receive VEVs $v_{d}$ and $v_{u}$, the singlet mixes with the other doublets ${ }^{3}$ In the CP-conserving case, this leads to three CP-even physical scalars and two $\mathrm{CP}$-odd ones together with the neutral would-be Goldstone boson. It is common to define the basis for the CP-even states as $\left(\phi_{d}, \phi_{u}, \phi_{s}\right)$ where $\phi_{i}$ are the real parts of the neutral components of $H_{d}, H_{u}$ and $S$. This leads to the tree-level mass matrix $\left(M_{h}^{2}\right)^{\text {tree }}$, derived from the tree-level scalar potential

$$
\left(M_{h}^{2}\right)_{i j}^{\text {tree }}=\frac{\partial^{2} V^{\text {tree }}}{\partial \phi_{i} \partial \phi_{j}} .
$$

The pole masses $m_{h_{i}}(i=1,2,3)$, identified with the masses of the three CP-even physical scalars, are the eigenvalues of the momentum dependent, loop-corrected mass matrix

$$
M_{h}^{2}\left(p^{2}\right)=\left(M_{h}^{2}\right)^{\text {tree }}+\sum_{n}^{\infty} \Pi^{(n)}\left(p^{2}\right),
$$

\footnotetext{
${ }^{3}$ While in the CP violating NMSSM the VEV s can be taken complex, it turns out that, after exploiting the minimum conditions of the scalar potential, the NMSSM Higgs sector at tree level can be described by one complex phase, which is given by $\varphi_{y}=\varphi_{\kappa}-\varphi_{\lambda}+2 \varphi_{s}-\varphi_{u}$, where $\varphi_{u}$ and $\varphi_{s}$ are the complex phases of the VEVs of $H_{u}$ and $S$, respectively, and $\varphi_{\kappa}$ and $\varphi_{\lambda}$ the complex phases of $\kappa$ and $\lambda$.
} 
each evaluated at $p^{2}=m_{h_{i}}^{2}$. The $\Pi^{(n)}\left(p^{2}\right)$ denote $3 \times 3$ matrices of $n$-loop self-energies calculated for an external momentum $p^{2}$. As mentioned in the introduction, only the one-loop contribution, $\Pi^{(1)}\left(p^{2}\right)$, is completely known so far. For $\Pi^{(2)}$ only partial results in the limit $p^{2}=0$ exist in the NMSSM. Different choices of the renormalization scheme lead to different results for the physical scalar masses if the perturbative series in eq. (9) is truncated at a given order $n$. The differences between the results are of $\mathcal{O}\left(\Pi^{(n+1)}\right)$, and provide an estimate of the theoretical uncertainty of the calculation due to uncomputed higher-order corrections. The matrix that diagonalizes $M_{h}^{2}\left(p^{2}\right)$ is called $Z^{H}$ in the following $4^{4}$

$$
m_{h}^{\text {diag }}=Z_{H} M_{h}^{2}\left(p^{2}\right) Z_{H}^{T} .
$$

In general, $Z_{H}$ is a $p^{2}$-dependent quantity. The NMSSM spectrum generators adopt different definitions for the loop-corrected rotation matrix $Z_{H}$ that they give as output: the external momentum is either set equal to zero, or set equal to one of the mass eigenvalues.

We conclude this section by presenting the set of input parameters that enter the calculation of the Higgs-boson masses and mixing matrices in the CP-conserving NMSSM. First, there are the three tadpole conditions that ensure the minimum of the Higgs potential at non-vanishing VEVs $v_{u}, v_{d}, v_{s}$,

$$
\left.\frac{\partial V^{\mathrm{eff}}}{\partial \phi_{i}}\right|_{\min }=0, \quad i=d, u, s,
$$

where $V^{\text {eff }}$ represents the effective scalar potential computed at the same perturbative order as the mass matrix in eq. (9). These conditions fix three parameters. The simplest choice is to solve them for $m_{s}^{2}, m_{H_{d}}^{2}$ and $m_{H_{u}}^{2}$, and this is done in all the comparisons for every code we use. Since one combination of VEVs (namely, $v^{2}=v_{u}^{2}+v_{d}^{2}$ ) is constrained by experimentally measured observables, this leaves six free parameters in the Higgs sector of the model:

$$
\lambda, \kappa, A_{\lambda}, A_{\kappa}, \tan \beta, \mu_{\mathrm{eff}},
$$

where $\tan \beta \equiv v_{u} / v_{d}$, and $\mu_{\text {eff }}$ stands in for $v_{s}$. Under the assumption that the soft SUSY-breaking terms are flavor-diagonal and that only third-generation soft interactions are non-negligible, this reduces the additional input parameters required to fully define the model to the set

$$
A_{t}, A_{b}, A_{\tau}, m_{\tilde{t}_{L}}, m_{\tilde{t}_{R}}, M_{1}, M_{2}, M_{3}, M_{\tilde{f}},
$$

with $\left.m_{\tilde{t}_{L}}^{2} \equiv m_{\tilde{q}, 33}^{2}, m_{\tilde{t}_{R}}^{2} \equiv m_{\tilde{u}, 33}^{2}\right\rfloor^{5}, A_{\tau}=A_{e, 33}, A_{b}=A_{d, 33}, A_{t}=A_{u, 33}$, and where we assumed for simplicity that all other diagonal entries of $m_{\tilde{f}}^{2}$ are degenerate and equal to $M_{\tilde{f}}^{2}$.

One can further reduce the number of input parameters by assuming a specific mechanism to transmit SUSY breaking from a hidden sector to our visible sector. In models inspired by minimal supergravity the following relations among the soft terms exist at the gauge-coupling unification scale:

$$
m_{\tilde{f}}^{2}=m_{0}^{2} \mathbf{1}, \quad M_{i}=M_{1 / 2}(i=1,2,3), \quad A_{j}=A_{0} \quad(j=e, d, u),
$$

where $\mathbf{1}$ is the identity matrix in flavor space. In contrast to the fully constrained NMSSM, the Higgs and singlet soft masses are obtained again by the tadpole equations, and also the NMSSM-specific trilinear soft couplings are treated as free parameters. Thus, necessary input parameters are

$$
m_{0}, M_{1 / 2}, \tan \beta, A_{0}, \lambda, \kappa, A_{\kappa}, A_{\lambda}, \mu_{e f f} .
$$

\section{Spectrum generators for the NMSSM and their features}

\subsection{Features}

The main features of the publicly available spectrum generators for the NMSSM are summarized in table 1. Here, two different kinds of spectrum generators are distinguished: dedicated tools which

\footnotetext{
${ }^{4}$ Note that, in eq. 10, , only one element of the matrix $m_{h}^{\text {diag }}$ at a time can correspond to a pole Higgs mass, and only for an appropriate choice of $p^{2}$.

${ }^{5}$ For further reference, we define also $m_{\tilde{u}_{R}}^{2} \equiv m_{\tilde{u}_{11}}^{2}, m_{\tilde{d}_{R}}^{2} \equiv m_{\tilde{d}_{11}}^{2}, m_{\tilde{u}_{L}}^{2} \equiv m_{\tilde{q}_{11}}^{2}$.
} 


\begin{tabular}{|c|c|c|c|c|c|}
\hline & FlexibleSUSY & NMSSMCALC & NMSSMTools & SOFTSUSY & SPheno \\
\hline \multicolumn{6}{|c|}{ Code } \\
\hline type & using SARAH & stand alone & stand alone & stand alone & using SARAH \\
\hline language & $\mathrm{C}++$ & $\begin{array}{l}\text { Fortran } 77 \\
\text { and } 90\end{array}$ & Fortran 77 & $\mathrm{C}++$ & Fortran 90 \\
\hline \multicolumn{6}{|c|}{ Models } \\
\hline No $\mathbb{Z}_{3}$ & $\sqrt{ }$ & $x$ & $\checkmark$ & $\checkmark$ & $\sqrt{ }$ \\
\hline GUT model & $\checkmark$ & $x$ & $\sqrt{ }$ & $\checkmark$ & $\sqrt{ }$ \\
\hline $\mathrm{CPV}$ & $(\sqrt{ })$ & $\sqrt{ }$ & $x$ & $x$ & $(\sqrt{ })$ \\
\hline \multicolumn{6}{|c|}{ Thresholds } \\
\hline scale(s) & $M_{Z}$ & $M_{t}, M_{S U S Y}$ & $M_{t}, M_{S U S Y}$ & $M_{Z}$ & $M_{Z}$ \\
\hline $\begin{array}{l}\text { EW parame- } \\
\text { ters }\end{array}$ & full one-loop & OS definitions & full one-loop & full one-loop & full one-loop \\
\hline Yukawas & $\begin{array}{l}\text { full one-loop; } \\
\text { two-loop QCD }\end{array}$ & $\begin{array}{c}\text { one-loop } \\
\text { (S)QCD; } \\
\text { two-loop QCD }\end{array}$ & $\begin{array}{l}\text { one-loop } \\
\text { (S)QCD+Yukawa; } \\
\text { two-loop QCD }\end{array}$ & $\begin{array}{l}\text { full one-loop; } \\
\text {; two-loop } \\
\text { QCD; } \\
\text { optionally } \\
\text { two-loop } \\
\text { SQCD }\end{array}$ & $\begin{array}{l}\text { full one-loop } \\
\text { two-loop QCD }\end{array}$ \\
\hline strong gauge & $\begin{array}{c}\text { one-loop } \\
\text { top+SUSY }\end{array}$ & - & - & $\begin{array}{l}\text { one-loop } \\
\text { top+SUSY }\end{array}$ & $\begin{array}{l}\text { one-loop } \\
\text { top+SUSY }\end{array}$ \\
\hline \multicolumn{6}{|c|}{ Higgs mass calculation } \\
\hline scheme & $\overline{\overline{\mathrm{DR}}}$ & $\overline{\mathrm{OS}} \overline{\overline{\mathrm{DR}}}$ & $\overline{\overline{\mathrm{DR}}}$ & $\overline{\overline{\mathrm{DR}}}$ & $\overline{\overline{\mathrm{DR}}}$ \\
\hline one-loop & full & full & full & full & full \\
\hline two-loop & $\begin{array}{l}\alpha_{s}\left(\alpha_{b}+\alpha_{t}\right) \\
+\operatorname{MSSM}\end{array}$ & $\alpha_{s} \alpha_{t}$ & $\begin{array}{c}\alpha_{s}\left(\alpha_{b}+\alpha_{t}\right) \\
+\operatorname{MSSM}\end{array}$ & $\begin{array}{l}\alpha_{s}\left(\alpha_{b}+\alpha_{t}\right) \\
\quad+\operatorname{MSSM}\end{array}$ & $\alpha_{s} \alpha_{i}+\alpha_{i} \alpha_{j}$ \\
\hline \multicolumn{6}{|c|}{ SUSY masses } \\
\hline one-loop & $\sqrt{ }$ & $x$ & $\checkmark$ & $\checkmark$ & $\sqrt{ }$ \\
\hline $\begin{array}{l}\text { momentum ef- } \\
\text { fects }\end{array}$ & $\checkmark$ & $x$ & $\begin{array}{l}\sqrt{ }\left(\alpha_{s}, \alpha_{t}, \alpha_{b}\right. \\
\quad \text { only })\end{array}$ & $\checkmark$ & $\checkmark$ \\
\hline \multicolumn{6}{|c|}{ Other observables } \\
\hline decays & $x$ & $\checkmark$ & via NMHDECAY & via NMHDECAY & $\checkmark$ \\
\hline flavour, $g-2$ & $x$ & $x$ & $\checkmark$ & $x$ & $\checkmark$ \\
\hline
\end{tabular}

Table 1: Public spectrum generators for the NMSSM and their features. The first two rows specify if the code is stand alone or gets necessary information from the Mathematica package SARAH, and in what language the code is written. The next three rows list the models that are supported: "GUT model" means that the code accepts as input a set of SUSYbreaking parameters at the GUT scale, and "CPV" shows if a Higgs sector with CP-even and -odd mixing is supported. Here, $(\checkmark)$ is used if this is only possible at the one-loop level so far. The rows under the sub-heading "Thresholds" give details of what SUSY and non-SUSY threshold corrections are included to calculate the running parameters entering the Higgs-mass calculation. The first row contains the scale (or scales) at which the threshold corrections are introduced, and the following three rows refer to different categories of parameters. Under "Higgs mass calculation" the main aspects of the calculation of the Higgs mass are given: the renormalization scheme ("scheme"), and what corrections at one- and two-loop are included. "full" at one-loop refers to a diagrammatic calculation including the entire $p^{2}$ dependence and all corrections up to those suppressed by first and second generation Yukawa couplings (which are also included in some codes). At two-loop the included corrections are shown. "+MSSM" refers to the approximation that the known MSSM results for the pure Yukawa interactions at two-loop are included. In the last column, $\alpha_{i}$ and $\alpha_{j}$ stand for any of the couplings $\alpha_{t}, \alpha_{b}, \alpha_{\tau}, \alpha_{\lambda}$ and $\alpha_{\kappa}$. Under "SUSY masses" it is shown if the code calculates also one-loop corrections to SUSY states (neutralinos, charginos, sfermions, gluino) and if the effects from the external momenta are included in these calculations. Finally, it is summarized in "Other observables" if the tool gives also a prediction for flavor observables and/or other precision observables like $g-2$ and if it calculates the decays of at least the Higgs bosons. 
contain all physically relevant information about the NMSSM (vertices, mass matrices, renormalization group equations (RGEs), loop corrections, etc.) in a hardcoded way, and multi-purpose generators which use the Mathematica package SARAH to derive this information for a given model. The advantage of the first approach, which is adopted by NMSSMTools, NMSSMCALC and SOFTSUSY, is that the tools can be used directly after compilation without further effort. In contrast, to use FlexibleSUSY and SPheno, one needs to first install SARAH 6 , which is then used to generate the necessary source code to get an NMSSM spectrum generator. These additional steps allow these tools to be used for a much broader range of models, i.e. not only other singlet extensions beyond the NMSSM are supported, but also many other extensions of the MSSM. Also, the features of the NMSSM version (like boundary conditions at all relevant scales) can be adjusted in the input files for SARAH or FlexibleSUSY without the need to modify the final code? SARAH is available at hepforge

$$
\text { http://sarah.hepforge.org/ }
$$

More details on the different tools are given in the following, in the order in which the codes became publicly available.

\subsubsection{NMSSMTools}

The first public codes to be made available for the NMSSM were NMHDECAY 42, 43, NMSPEC [44] and NMGMSB, which are today collected in the package NMSSMTools. NMHDECAY was based on a suitably modified version of HDECAY [63, 64, 65]. This package is available at

$$
\text { http://www.th.u-psud.fr/NMHDECAY/nmssmtools.html }
$$

So far NMSSMTools is restricted to the real NMSSM with and without $\mathbb{Z}_{3}$ symmetry, but a version to support also CP violation is under construction [66]. NMSSMTools allows to calculate the Higgs masses for a parameter point with three different options:

1. The most precise calculation makes use of the NMSSM corrections of ref. [36. This provides a fully diagrammatic calculation of the Higgs masses at the one-loop level, including all contributions and the momentum dependence, plus the two-loop corrections of $\mathcal{O}\left(\alpha_{s}\left(\alpha_{b}+\alpha_{t}\right)\right)$ at zero external momentum. In addition, the two-loop corrections known from the MSSM at $\mathcal{O}\left(\left(\alpha_{t}+\alpha_{b}\right)^{2}+\alpha_{\tau}\left(\alpha_{\tau}+\alpha_{b}\right)\right)$ [67, 68, 69, 57] are included.

2. The second option implements the same corrections as the first one, but neglects the momentum dependence of the one-loop self-energies. (This makes the code faster, which is useful for large scans.)

3. The third option is the original implementation of the Higgs mass calculation in NMSSMTools, which - as described in the appendix C of ref. [9] - implements only a partial computation of the one-loop contributions, and restricts the two-loop contributions to the leading-log approximation.

NMSSMTools also calculates the SUSY spectrum at the one-loop level, and the Higgs and sparticle decay branching fractions (the latter in NMSDECAY [70] using a suitable modification of SDECAY [71]). $B$-physics observables and the muon anomalous magnetic moment are computed following refs. [72, 73], and compared to experimental constraints. Bounds on the Higgs sector from LEP [74] and on the couplings of the SM-like Higgs boson (from ref. [75]) are implemented. A link to MicrOmegas is included which allows for an easy calculation of the dark matter relic density, and direct and indirect dark matter detection cross sections.

It is possible to define a set of input parameters at the SUSY scale, or to define specific SUSYbreaking scenarios such as minimal supergravity using NMSPEC, or scenarios of gauge-mediated SUSY breaking (following ref. [76]) using NMGMSB. In all cases, the fine-tuning of the EWSB scale with respect to the input parameters can be estimated.

\footnotetext{
${ }^{6}$ FlexibleSUSY also offers pre-generated code for selected models, with specific boundary conditions and EWSB outputs, allowing one to use these specific FlexibleSUSY spectrum generators without first installing Mathematica and SARAH.

${ }^{7}$ For easier comparison with other codes, we have created input files to allow for purely SLHA1 61] input for SPheno and FlexibleSUSY, while by default a block form for all matrices is used as in SLHA2 62. Interested readers can obtain the files from the authors.
} 


\subsubsection{SPheno and SARAH}

The main features of the SPheno version generated with SARAH for the NMSSM are a precise massspectrum calculation using the full two-loop RGEs of the NMSSM, with all flavor and CP effects, for the running between $M_{Z}, M_{S U S Y}$ and $M_{G U T}$. The RGEs are calculated using generic expressions given in Refs. [77, 78, 79, 80, 81, 82, 83, 84. SPheno calculates also the full one-loop corrections to all masses, including the entire momentum dependence in the $\overline{\mathrm{DR}}$ scheme. At two-loop all corrections in the gaugeless limit with vanishing external momenta are included [85, 86], by making use of generic expressions given in Refs. [87, 88, 89. This approach makes it possible to include all twoloop corrections without making use of any MSSM approximations as done by other codes, i.e. also the NMSSM-specific corrections of $\mathcal{O}\left(\alpha_{\lambda}\left(\alpha_{\lambda}+\alpha_{\kappa}+\alpha_{t}\right)\right)$ are fully included. However, it is optionally also possible to make use of results from the literature for the two-loop corrections in the MSSM and NMSSM. These routines are usually much faster but have to be used carefully for models beyond the MSSM. Higgs sectors with CP violation are so far supported only at the one-loop level.

Other features of the SPheno NMSSM version are the calculation of all important quark-flavor violating observables ( $B$ and $K$ decays, $\Delta M_{B_{d, s}} / \Delta M_{K}, \epsilon_{K}$ ) as well as lepton-flavor violating observables using the FlavorKit functionality 90. Also, $(g-2)_{l}$ and electromagnetic dipole moments are predicted. Moreover, all two- and three-body decays of SUSY particles, and two-body decays of the Higgs scalars are calculated. The sparticle decays are purely tree-level, while for the Higgs bosons the NLO-QCD corrections to decays in two quarks, photons and gluons are included. Also decays in virtual vector bosons are taken into account. Finally, SPheno can make a prediction for the electroweak fine-tuning according to measures proposed in Refs. [91, 92] and writes all necessary input files to test points with HiggsBounds 93, 94, 95, and HiggsSignals 96.

The basic SPheno version that is used together with SARAH can be downloaded from

$$
\text { http://spheno.hepforge.org/ }
$$

A handy possibility to interface SPheno versions created by SARAH with Monte-Carlo tools like MadGraph [97, 98] and WHIZARD [99, 100] as well as MicrOmegas is available via the BSM Toolbox [101.

\subsubsection{NMSSMCALC}

NMSSMCALC allows for the computation of the Higgs masses both in the CP-conserving and CPviolating NMSSM, both at one-loop and at two-loop level. In contrast to the other codes, NMSSMCALC makes use of mixed $\overline{\mathrm{DR}}-\mathrm{OS}$ renormalization conditions for the computation of the Higgs masses. In the (s)top sector the user can choose between OS and $\overline{\mathrm{DR}}$ renormalization. In addition, if the charged Higgs boson mass $M_{H^{ \pm}}$is given as an input instead of $A_{\lambda}, M_{H^{ \pm}}$enters the Higgs mass calculation as an OS parameter. For comparison between the codes, however, the option of $\overline{\mathrm{DR}}$ renormalization of the (s)top sector and the option with the input $A_{\lambda}$ are chosen. The Higgs mass calculation at one-loop level is performed including the full momentum dependence and all possible contributions [38, 39]. At the two-loop level the $\mathcal{O}\left(\alpha_{s} \alpha_{t}\right)$ corrections are included [41. They include the $\mathcal{O}\left(\alpha_{s} \alpha_{t}\right)$ part relating the vacuum expectation value to physical observables, which is missing so far in the other spectrum generators.

NMSSMCALC can be downloaded from

$$
\text { http://www.itp.kit.edu/ maggie/NMSSMCALC/ }
$$

and comes together with an NMSSM extension of HDECAY [63, 64, 65] for the Higgs boson decays. The decays are calculated in the CP-conserving and CP-violating NMSSM. The decay widths include the dominant higher-order QCD corrections. Furthermore, the decays of the neutral Higgs bosons into a bottom pair include the higher order SUSY-QCD and the approximate SUSY-electroweak (EW) corrections up to one-loop order. The decays into a strange quark pair include the dominant resummed SUSY-QCD and the ones into $\tau$ pairs the dominant resummed SUSY-EW corrections. Accordingly the charged Higgs boson decays into fermion pairs include the higher order SUSY corrections. All two-body decays into SUSY particles have been implemented. For the CP-conserving case the decays into stop and sbottom pairs come with the SUSY-QCD corrections. Finally, all relevant off-shell decays into two massive gauge boson final states, into gauge and Higgs boson final states, into Higgs pairs as well as into heavy quark pairs are included. For more details and a complete list of references see 48. 


\subsubsection{SOFTSUSY}

SOFTSUSY is a widely used spectrum generator for the MSSM with and without $R$-parity violation which has recently been extended to the NMSSM. The NMSSM spectrum generator is implemented to solve scenarios with and without the $\mathbb{Z}_{3}$ symmetry. The implementation of EWSB conditions allows one to choose as output for the $\mathbb{Z}_{3}$-conserving case either the set $\left\{\kappa, v_{s}, m_{s}^{2}\right\}$ or the set $\left\{m_{H_{u}}^{2}, m_{H_{d}}^{2}, m_{s}^{2}\right\}$, and in the $\mathbb{Z}_{3}$-violating case there is an additional option of giving out $\left\{\mu, B_{\mu}, \chi_{s}\right\}$. For the highscale boundary conditions there are a number of pre-defined options including the mSUGRA-inspired semi-constrained NMSSM and a general high-scale boundary condition which allows one to set all soft SUSY-breaking parameters independently. The users may also easily create their own boundary conditions.

For the NMSSM, SOFTSUSY extends the full three-family one- and two-loop RGEs of the MSSM [77, 102 using general two-loop expressions from the literature [77, 78, with the NMSSM specialization given in Appendix D of the NMSSM manual [46]. These expressions were also cross-checked against two-loop NMSSM RGEs in the literature that use the 3rd-family approximation [9], and tested numerically against the RGEs from SARAH using an early development code from FlexibleSUSY. Finally, the RGEs for the vacuum expectation values are implemented using Refs. [83, 84. The one-loop self-energies and tadpole corrections for the Higgs bosons were extended to the NMSSM using the expressions in Refs. [36, 37]. The NMSSM extension also includes the two-loop corrections at order $\mathcal{O}\left(\alpha_{s}\left(\alpha_{t}+\alpha_{b}\right)\right)$ from Ref. [36] and in addition uses the MSSM results from Refs. [67, 68, 69, 57.

Higgs and sparticle decays may be obtained by interfacing SoftSUSY with NMHDECAY [42, 43 , and NMSDECAY [70, which is based upon Ref. [71. These are both distributed as part of the NMSSMTools package, and SOFTSUSY provides a script to do the interface with this package automatically.

The homepage of SOFTSUSY is

$$
\text { http://softsusy.hepforge.org/ }
$$

\subsubsection{FlexibleSUSY}

FlexibleSUSY is a Mathematica and $\mathrm{C}++$ package, which creates spectrum generators for userdefined (SUSY or non-SUSY) models. It makes use of SARAH to obtain model-dependent details, i.e. couplings, RGEs, self-energies and tadpole corrections. The boundary conditions on the model parameters at different scales, as well as spectrum-generator specific configuration details are specified in a FlexibleSUSY model file. FlexibleSUSY can therefore generate spectrum generators for the different MSSM singlet extensions provided by SARAH, for example the NMSSM with and without a $\mathbb{Z}_{3}$ symmetry. The generated spectrum generators consist of $\mathrm{C}++$ classes, which can be easily adapted allowing one to use the routines in a custom-made program for solving non-standard problems. For example one may build a tower of effective field theories, which could be useful for NMSSM scenarios with very heavy superparticles. Currently, FlexibleSUSY fully supports only real parameters. Complex parameters have been developed very recently in version 1.1.0, but they are still undocumented and undergoing testing. The automatic implementation of alternative boundary-value-problem solvers and automatic generation of towers of effective field theories are currently under development. Additionally, an extension to calculate decays, FlexibleDecay, for both Higgs and superparticles is currently being developed.

Due to the use of SARAH, FlexibleSUSY employs the full two-loop RGEs and one-loop corrections to the pole mass spectrum. At the two-loop level it makes use of Higgs mass corrections available in the literature. In the case of the NMSSM FlexibleSUSY uses the $\alpha_{s}\left(\alpha_{t}+\alpha_{b}\right)$ corrections given in Ref. [36]. In addition, the MSSM two-loop corrections based on Refs. [67, 68, 69, 57, are used, though the user may choose to switch any of these two-loop corrections off using the SLHA file.

The homepage of FlexibleSUSY is

$$
\text { http://flexiblesusy.hepforge.org/ }
$$




\subsection{Used codes and options}

For the comparison in the following, these versions of the different codes were used:

- NMSSMTools 4.7.0

- SPheno 3.3.6 together with SARAH 4.5.7

- NMSSMCALC 1.0 .3

- SOFTSUSY 3.6 .0

- FlexibleSUSY 1.1 .0 together with SARAH 4.5.7

We always make use of the most precise calculation available in each code, i.e. all available two-loop corrections turned on and in NMSSMTools we only compare Higgs masses calculated using option 1, described in section 3.1.1 and do not consider any of the approximations from alternative options. We also concentrate on calculations done in the $\overline{\mathrm{DR}}$ scheme. A discussion of the effects using an OS scheme is beyond the scope of this comparison and will be given elsewhere including also results from the upcoming NMSSM version of FeynHiggs [103, 104, 105, 106, 107].

\subsection{Predictions for the CP-even and CP-odd Higgs masses}

We discuss here the Higgs mass predictions of the various codes for six different test points (TP) with the following features:

- MSSM-like point (TP1)

- MSSM-like point with large stop splitting (TP2)

- Point with light singlet and $\lambda$ close to the perturbativity limit (TP3)

- Point with heavy singlet and $\lambda$ close to the perturbativity limit (TP4)

- Point with slightly lighter singlet. Additional matter is needed for perturbativity; inspired by NMP9 [108] (TP5)

- Point with huge $\lambda$ (TP6)

In table 2 we list the input parameters that are different for the six benchmark points. As is usual in NMSSM studies, for the last four points we choose smallish values of $\tan \beta$, to avoid suppressing the NMSSM-specific contributions to the SM-like Higgs mass. For very large values of $\tan \beta$ additional differences between the codes are expected, since not all of them include the two-loop corrections involving the bottom and $\tau$ Yukawa coupling.

All values in this table apart from the one for $\tan \beta$ are defined at the scale $Q$. The shown value for $\tan \beta$ refers to the scale $M_{Z} 8$ The remaining soft SUSY-breaking parameters, common to all points, are

$$
M_{\tilde{f}}=1500 \mathrm{GeV}, A_{\tau}=0 .
$$

In addition, if possible via the input file, the codes were forced to calculate the masses directly at the input scale $Q$. This is possible for all tools except NMSSMTools. More details about the general handling of the scales in the different codes will be given in section 4.1.3. These points are chosen in a way that differences between the predicted Higgs masses are visible. However, they are not the result of an exhaustive scan to find points with the largest possible differences. Thus, we expect that these points are representative of a non-negligible fraction of the parameter space of the NMSSM.

\footnotetext{
${ }^{8}$ Since NMSSMCALC and NMSSMTools expect $\tan \beta$ to be defined at $Q$ as well, the input values have to be adjusted accordingly.
} 


\begin{tabular}{|c|c|cccccc|ccccccc|}
\hline & $Q$ & $\tan \beta$ & $\lambda$ & $\kappa$ & $A_{\lambda}$ & $A_{\kappa}$ & $\mu_{\text {eff }}$ & $M_{1}$ & $M_{2}$ & $M_{3}$ & $A_{t}$ & $A_{b}$ & $m_{\tilde{t}_{L}}$ & $m_{\tilde{t}_{R}}$ \\
\hline TP1 & 1500 & 10 & 0.1 & 0.1 & -10 & -10 & 900 & 500 & 1000 & 3000 & 3000 & 0 & 1500 & 1500 \\
TP2 & 1500 & 10 & 0.05 & 0.1 & -200 & -200 & 1500 & 1000 & 2000 & 2500 & -2900 & 0 & 2500 & 500 \\
TP3 & 1000 & 3 & 0.67 & 0.1 & 650 & -10 & 200 & 200 & 400 & 2000 & 1000 & 1000 & 1000 & 1000 \\
TP4 & 750 & 2 & 0.67 & 0.2 & 405 & 0 & 200 & 120 & 200 & 1500 & 1000 & 1000 & 750 & 750 \\
TP5 & 1500 & 3 & 0.67 & 0.2 & 570 & -25 & 200 & 135 & 200 & 1400 & 0 & 0 & 1500 & 1500 \\
TP6 & 1500 & 3 & 1.6 & 1.61 & 375 & -1605 & 614 & 200 & 400 & 2000 & 0 & 0 & 1500 & 1500 \\
\hline
\end{tabular}

Table 2: Input parameters for the test points. All dimensionful parameters in units of GeV.

TP1 and TP2 have small couplings between the Higgs doublets and the singlet. Thus, a mass of about $125 \mathrm{GeV}$ for the SM-like state can only be obtained via large (s)top corrections as in the MSSM. In contrast, TP3-TP6 come with a large $\lambda$ coupling and a small value for $\tan \beta$. This already increases the mass of the SM-like scalar at tree level. However, additional loop corrections beyond the MSSM are also expected to become more important. The difference between these four points is that TP3 and TP4 have values for $\lambda$ and $\kappa$ that give a consistent model without any Landau pole up to the GUT scale of $10^{16} \mathrm{GeV}$. For TP5 $\lambda$ becomes non-perturbative below the GUT scale unless additional matter is assumed to change the running. TP6 has a huge $\lambda$ which always leads to a cut-off. Scenarios with these large $\lambda$ couplings can be motivated by naturalness considerations [109].

\begin{tabular}{|l|cccccc|}
\hline & TP1 & TP2 & TP3 & TP4 & TP5 & TP6 \\
\hline & \multicolumn{7}{|c|}{$h_{1}$} \\
\hline FlexibleSUSY & $\mathbf{1 2 3 . 5 5}$ & $\mathbf{1 2 2 . 8 4}$ & 91.11 & $\mathbf{1 2 7 . 6 2}$ & 120.86 & $\mathbf{1 2 6 . 4 6}$ \\
NMSSMCALC & $\mathbf{1 2 0 . 3 4}$ & $\mathbf{1 1 8 . 5 7}$ & 90.88 & $\mathbf{1 2 6 . 3 7}$ & 120.32 & $\mathbf{1 2 3 . 4 5}$ \\
NMSSMTOOLS & $\mathbf{1 2 3 . 5 2}$ & $\mathbf{1 2 1 . 8 3}$ & 90.78 & $\mathbf{1 2 7 . 3 0}$ & 119.31 & $\mathbf{1 2 6 . 6 3}$ \\
SOFTSUSY & $\mathbf{1 2 3 . 8 4}$ & $\mathbf{1 2 3 . 0 8}$ & 90.99 & $\mathbf{1 2 7 . 5 2}$ & 120.81 & $\mathbf{1 2 6 . 6 7}$ \\
SPHENO & $\mathbf{1 2 4 . 8 4}$ & $\mathbf{1 2 4 . 7 4}$ & 89.54 & $\mathbf{1 2 6 . 6 2}$ & 119.11 & $\mathbf{1 3 1 . 2 9}$ \\
\hline & \multicolumn{6}{|c|}{$h_{2}$} \\
\hline FlexibleSUSY & 1797.46 & 5951.36 & $\mathbf{1 2 6 . 5 8}$ & 143.11 & $\mathbf{1 2 5 . 0 8}$ & 700.80 \\
NMSSMCALC & 1797.46 & 5951.36 & $\mathbf{1 2 4 . 8 6}$ & 142.59 & $\mathbf{1 2 3 . 1 4}$ & 701.02 \\
NMSSMTOOLS & 1797.46 & 5951.36 & $\mathbf{1 2 7 . 2 8}$ & 144.07 & $\mathbf{1 2 6 . 9 5}$ & 700.46 \\
SOFTSUSY & 1797.46 & 5951.36 & $\mathbf{1 2 6 . 5 9}$ & 143.02 & $\mathbf{1 2 5 . 1 2}$ & 701.01 \\
SPHENO & 1798.01 & 5951.35 & $\mathbf{1 2 6 . 7 7}$ & 144.04 & $\mathbf{1 2 5 . 6 1}$ & 689.30 \\
\hline & \multicolumn{7}{|c|}{$h_{3}$} \\
\hline FlexibleSUSY & 2758.96 & 6372.08 & 652.95 & 467.80 & 627.28 & 1369.53 \\
NMSSMCALC & 2756.37 & 6371.31 & 652.48 & 467.42 & 627.00 & 1368.68 \\
NMSSMTOOLS & 2758.51 & 6345.72 & 651.03 & 466.38 & 623.79 & 1368.90 \\
SOFTSUSY & 2758.41 & 6370.3 & 652.78 & 467.73 & 627.14 & 1369.19 \\
SPHENO & 2757.11 & 6366.88 & 651.21 & 467.5 & 624.02 & 1363.02 \\
\hline \hline
\end{tabular}

Table 3: Masses for the CP-even scalars (in GeV) for TP1-TP6 when using the spectrum generators "out-of-the-box". The values correspond to the two-loop results obtained by the different tools. For NMSSMCALC the value using the $\overline{\mathrm{DR}}$ scheme is shown and for NMSSMTools the value using the most precise calculation is given. The masses for the SM-like scalar are written in bold fonts, those for the singlet-like scalar in italics.

The scalar masses calculated for these six points by the five spectrum generators as they come "out-of-the-box" are listed in table 3 , and the absolute values of the entries of the rotation matrix $Z_{H}$ that correspond to the SM-like scalar are listed in tables 4 45 . For TP1, TP2 and TP6 the singlet-like scalar is very heavy and decouples, for TP4 the singlet-like scalar is just slightly heavier than the SM-like scalar, while for TP3 and TP5 it is the lightest scalar. We find that, even for the MSSM-like points, the differences in the mass of the SM-like scalar can exceed $5 \mathrm{GeV}$. For the points with very large $\lambda$ the difference can be even larger. The masses for the other two scalars usually agree quite well between the codes. Only SPheno gives masses of the singlet-like states, for TP5 and TP6, which differ by $1-2 \%$ compared to all other tools. 


\begin{tabular}{|l|ccc|ccc|ccc|}
\hline & \multicolumn{3}{|c|}{ TP1 } & \multicolumn{3}{c|}{ TP2 } & \multicolumn{3}{c|}{ TP3 } \\
& $\left|Z_{i 1}\right|$ & $\left|Z_{i 2}\right|$ & $\left|Z_{i 3}\right|$ & $\left|Z_{i 1}\right|$ & $\left|Z_{i 2}\right|$ & $\left|Z_{i 3}\right|$ & $\left|Z_{i 1}\right|$ & $\left|Z_{i 2}\right|$ & $\left|Z_{i 3}\right|$ \\
\hline FlexibleSUSY & 0.1039 & 0.9946 & 0.0076 & 0.1034 & 0.9946 & 0.0004 & 0.2172 & 0.1888 & 0.9577 \\
NMSSMCALC & 0.1039 & 0.9946 & 0.0076 & 0.1034 & 0.9946 & 0.0004 & 0.2177 & 0.1923 & 0.9569 \\
NMSSMTOOLS & 0.1039 & 0.9946 & 0.0076 & 0.1038 & 0.9946 & 0.0004 & 0.2229 & 0.2115 & 0.9511 \\
SOFTSUSY & 0.1039 & 0.9946 & 0.0076 & 0.1034 & 0.9946 & 0.0004 & 0.2164 & 0.1883 & 0.958 \\
SPHENO & 0.104 & 0.9945 & 0.0074 & 0.1035 & 0.9946 & 0.0004 & 0.2269 & 0.2265 & 0.9472 \\
\hline FlexibleSUSY & 0.0075 & 0.0069 & 0.9999 & 0.0096 & 0.0006 & 1. & 0.2814 & 0.9274 & 0.2466 \\
NMSSMCALC & 0.0075 & 0.0068 & 0.9999 & 0.0095 & 0.0006 & 1. & 0.2811 & 0.9265 & 0.2502 \\
NMSSMTOOLS & 0.0076 & 0.0069 & 0.9999 & 0.0101 & 0.0006 & 0.9999 & 0.2782 & 0.9216 & 0.2708 \\
SOFTSUSY & 0.0075 & 0.0068 & 0.9999 & 0.0096 & 0.0006 & 1. & 0.282 & 0.9273 & 0.246 \\
SPHENO & 0.0075 & 0.0067 & 0.9999 & 0.0096 & 0.0006 & 1. & 0.2725 & 0.919 & 0.2851 \\
\hline FlexibleSUSY & 0.9946 & 0.1039 & 0.0068 & 0.9946 & 0.1034 & 0.0096 & 0.9347 & 0.3231 & 0.1483 \\
NMSSMCALC & 0.9946 & 0.104 & 0.0068 & 0.9946 & 0.1034 & 0.0095 & 0.9347 & 0.3234 & 0.1476 \\
NMSSMTOOLS & 0.9946 & 0.104 & 0.0068 & 0.9945 & 0.1038 & 0.0102 & 0.9338 & 0.3255 & 0.1484 \\
SOFTSUSY & 0.9946 & 0.104 & 0.0068 & 0.9946 & 0.1034 & 0.0096 & 0.9347 & 0.3234 & 0.1476 \\
SPHENO & 0.9945 & 0.104 & 0.0068 & 0.9946 & 0.1035 & 0.0097 & 0.935 & 0.3228 & 0.1468 \\
\hline
\end{tabular}

Table 4: Absolute values of the entries of the rotation matrix $Z_{H}$ corresponding to the SM-like scalar for TP1-TP3 when using the spectrum generators "out-of-the-box". The values correspond to the two-loop results obtained by the different tools. For NMSSMCALC the value using the $\overline{\mathrm{DR}}$ scheme is shown and for NMSSMTools the value using the most precise calculation is given.

\section{Breaking down the differences}

\subsection{Main differences}

The differences we have observed are larger than one usually finds for the MSSM when comparing the widely used tools that perform a $\overline{\mathrm{DR}}$ calculation [57, 58]. We pinned down four sources which will be discussed in the following. The numerical impact of these differences is discussed in sec. 4.4. Further differences, such as e.g. whether first and second generation fermions are included into the one-loop computation (NMSSMCALC, SPheno, FlexibleSUSY) or not (NMSSMTools, SOFTSUSY), are numerically tiny.

\subsubsection{Renormalization scheme}

All codes but NMSSMCALC perform a pure $\overline{\mathrm{DR}}$ renormalization. NMSSMCALC uses instead mixed $\overline{\mathrm{DR}}-$ OS renormalization conditions at one-loop level. At two-loop level it offers an option to renormalize the (s)top sector not only OS but also $\overline{\mathrm{DR}}$.

\subsubsection{Calculation of the running parameters}

A very crucial point is how the $\overline{\mathrm{DR}}$ parameters for the gauge and Yukawa couplings at the SUSY scale are calculated, since these couplings affect all loop calculations.

Top Yukawa coupling. The most important parameter entering the loop calculations in the Higgs sector is the top Yukawa coupling. To calculate the value of the $\overline{\mathrm{DR}}$-renormalized Yukawa coupling at the SUSY scale, there are two different approaches implemented in the codes:

1. SUSY thresholds at $M_{Z}:$ FlexibleSUSY, SOFTSUSY and SPheno adapt to the NMSSM the approach presented in Ref. [110] to calculate the running parameters of the MSSM Lagrangian at the SUSY scale. In this setup all one-loop SUSY and non-SUSY thresholds are calculated at the weak scale, identified with the $Z$-boson mass $M_{Z}$, and also the known two-loop QCD corrections of [111, 112] are included. The evolution of the parameters from $M_{Z}$ to the SUSY scale $M_{S U S Y}$ is done using two-loop SUSY RGEs. A detailed description of the calculation is given in Ref. [49] for FlexibleSUSY, Ref. [46] for SOFTSUSY and in Ref. [113] for SPheno. All three codes use in principle the same approach: 


\begin{tabular}{|l|ccc|ccc|ccc|}
\hline & \multicolumn{3}{|c|}{ TP4 } & \multicolumn{3}{c|}{ TP5 } & \multicolumn{3}{c|}{ TP6 } \\
& $\left|Z_{i 1}\right|$ & $\left|Z_{i 2}\right|$ & $\left|Z_{i 3}\right|$ & $\left|Z_{i 1}\right|$ & $\left|Z_{i 2}\right|$ & $\left|Z_{i 3}\right|$ & $\left|Z_{i 1}\right|$ & $\left|Z_{i 2}\right|$ & $\left|Z_{i 3}\right|$ \\
\hline FlexibleSUSY & 0.4866 & 0.7908 & 0.3712 & 0.2867 & 0.4021 & 0.8695 & 0.2741 & 0.9417 & 0.195 \\
NMSSMCALC & 0.4869 & 0.7849 & 0.3832 & 0.2967 & 0.4433 & 0.8459 & 0.2746 & 0.9416 & 0.1947 \\
NMSSMTOOLS & 0.4855 & 0.7495 & 0.4501 & 0.3072 & 0.4711 & 0.8269 & 0.2743 & 0.9416 & 0.1953 \\
SOFTSUSY & 0.4867 & 0.7902 & 0.3725 & 0.2863 & 0.402 & 0.8697 & 0.2747 & 0.9417 & 0.1941 \\
SPHENO & 0.4835 & 0.7646 & 0.4262 & 0.3033 & 0.4703 & 0.8288 & 0.2712 & 0.9407 & 0.2039 \\
\hline FlexibleSUSY & 0.0370 & 0.4059 & 0.9132 & 0.2189 & 0.8561 & 0.4681 & 0.2927 & 0.1115 & 0.9497 \\
NMSSMCALC & 0.0447 & 0.4158 & 0.9084 & 0.2055 & 0.8354 & 0.5099 & 0.2919 & 0.1113 & 0.9500 \\
NMSSMTOOLS & 0.0834 & 0.4727 & 0.8772 & 0.1974 & 0.8184 & 0.5396 & 0.2937 & 0.1114 & 0.9494 \\
SOFTSUSY & 0.0385 & 0.4066 & 0.9128 & 0.2195 & 0.8561 & 0.4679 & 0.2918 & 0.1107 & 0.9501 \\
SPHENO & 0.0682 & 0.4525 & 0.8892 & 0.1958 & 0.8204 & 0.5372 & 0.2945 & 0.1206 & 0.948 \\
\hline FlexibleSUSY & 0.8728 & 0.4581 & 0.1683 & 0.9327 & 0.3245 & 0.1575 & 0.9161 & 0.3174 & 0.2451 \\
NMSSMCALC & 0.8723 & 0.4594 & 0.1674 & 0.9326 & 0.3251 & 0.1568 & 0.9162 & 0.3177 & 0.2443 \\
NMSSMTOOLS & 0.8703 & 0.4634 & 0.1670 & 0.9309 & 0.3291 & 0.1584 & 0.9157 & 0.3177 & 0.2460 \\
SOFTSUSY & 0.8727 & 0.4587 & 0.1675 & 0.9327 & 0.3249 & 0.1568 & 0.9162 & 0.3176 & 0.2444 \\
SPHENO & 0.8727 & 0.4589 & 0.1667 & 0.9326 & 0.3252 & 0.1567 & 0.9164 & 0.3172 & 0.2443 \\
\hline
\end{tabular}

Table 5: The same as Tab. 5 for TP4-TP6.

the $\overline{\mathrm{DR}}$ mass of the top quark is computed as

$$
m_{t}^{\overline{\mathrm{DR}}}=m_{t}^{\text {pole }}+\hat{m}_{t} \Sigma\left(\hat{m}_{t}^{2}\right),
$$

where $\Sigma\left(p^{2}\right)$ is the (dimensionless) self-energy for momentum $p !^{9}$ For other fermions, the relation becomes more complicated because of the $\tan \beta$ resummation which is performed by the codes. The $\overline{\mathrm{DR}}$ Yukawa couplings are then computed at $M_{Z}$ by dividing the $\overline{\mathrm{DR}}$ fermion masses by the appropriate VEVs. There is however a difference between the codes in the definition of the mass $\hat{m}_{t}$ entering the correction in eq. (17): SOFTSUSY and FlexibleSUSY use $\hat{m}_{t}=m_{t}^{\text {pole }}$, while SPheno uses $\hat{m}_{t}=m_{t}^{\overline{\mathrm{DR}}}$. The effect of the different definition of $\hat{m}_{t}$ in the one-loop part of correction is partially compensated for by an appropriate shift in the two-loop QCD part of the correction.

2. SUSY thresholds at $M_{S U S Y}$ : In this scheme, the pole top mass is converted to the $\overline{\mathrm{MS}}$ scheme at the scale $m_{t}$, and the RGEs of the SM are used for the running to $M_{S U S Y}$. At $M_{S U S Y}$ the conversion of the $\overline{\mathrm{MS}}$ top mass of the SM to the corresponding $\overline{\mathrm{DR}}$ Yukawa coupling of the NMSSM takes place including the SUSY thresholds. This is the approach followed in NMSSMTOols and NMSSMCALC. However, there remain some differences in the concrete realization. NMSSMCALC uses the NLO-QCD contributions to the top-mass RGE for the running up to $M_{S U S Y}$, and includes only the SQCD threshold corrections at $M_{S U S Y}$. NMSSMTools resums also large logarithms $\sim Y_{t}^{2} \log \left(M_{S U S Y} / m_{t}\right)$ and $\sim Y_{t}^{2} \log \left(M_{S U S Y} / M_{A}\right)$ (the latter in case the scale $M_{A}$ of the heavy MSSM-like scalars differs from the scale $M_{S U S Y}$ of the stop/sbottom) and it includes also $\mathcal{O}\left(\alpha_{t}\right)$ threshold corrections from stop-Higgsino loops at $M_{S U S Y}$.

Strong interaction coupling. The strong interaction coupling at the scale $M_{S U S Y}$, important for the two-loop corrections to the Higgs masses, is derived as follows:

\footnotetext{
${ }^{9}$ More precisely, SPheno calculates the chiral and scalar self-energy contributions $\Sigma_{L}, \Sigma_{R}, \Sigma_{S}$ to the $3 \times 3$ mass matrix, but this only leads to differences if flavor violation is included.
} 
- FlexibleSUSY, SOFTSUSY, SPheno calculate $\alpha_{s}^{\overline{\mathrm{DR}}}\left(M_{Z}\right)$ via

$$
\begin{gathered}
\alpha_{s}^{\overline{\mathrm{DR}}}\left(M_{Z}\right)=\frac{\alpha_{s}^{(5), \overline{\mathrm{MS}}}\left(M_{Z}\right)}{1-\Delta \alpha_{s}\left(M_{Z}\right)}, \\
\Delta \alpha_{s}\left(M_{Z}\right)=\frac{\alpha_{s}}{2 \pi}\left(\frac{1}{2}-2 \log \frac{m_{\tilde{g}}}{M_{Z}}-\frac{2}{3} \log \frac{m_{t}}{M_{Z}}-\frac{1}{6} \sum_{i=1}^{6}\left(\log \frac{m_{\tilde{u}_{i}}}{M_{Z}}+\log \frac{m_{\tilde{d}_{i}}}{M_{Z}}\right)\right),
\end{gathered}
$$

where $\alpha_{s}^{(5), \overline{\mathrm{MS}}}$ denotes the strong coupling constant of the SM with $N_{f}=5$ active flavors. Finally, $\alpha_{s}^{\overline{\mathrm{DR}}}\left(M_{Z}\right)$ is run to $M_{S U S Y}$ using the two-loop RGEs of the NMSSM.

- NMSSMCALC: $\alpha_{s}^{\overline{\mathrm{DR}}}\left(M_{S U S Y}\right)$ is computed by running $\alpha_{s}^{(5), \overline{\mathrm{MS}}}\left(M_{Z}\right)$ up to the top pole mass $m_{t}$ using the QCD part of the two-loop RGEs of the SM with $N_{f}=5$ active flavors. Afterwards, the so-obtained $\alpha_{s}^{\overline{\mathrm{MS}}}\left(m_{t}\right)$ is run to $M_{S U S Y}$ using the corresponding RGEs with $N_{f}=6$ active flavors. With this procedure the one-loop top threshold correction for $\alpha_{s}^{\overline{\mathrm{MS}}}$ is taken into account implicitly. Finally, $\alpha_{s}^{\overline{\mathrm{MS}}}\left(M_{S U S Y}\right)$ is converted to $\overline{\mathrm{DR}}$ by

$$
\alpha_{s}^{\overline{\mathrm{DR}}}\left(M_{S U S Y}\right)=\frac{\alpha_{s}^{\overline{\mathrm{MS}}}\left(M_{S U S Y}\right)}{1-\frac{\alpha_{s}^{\overline{\mathrm{MS}}}\left(M_{S U S Y}\right)}{4 \pi}} .
$$

- NMSSMTools: $\alpha_{s}^{\overline{\mathrm{MS}}}\left(M_{S U S Y}\right)$ is computed with a procedure analogous to the one of NMSSMCALC, but it is not converted to the $\overline{\mathrm{DR}}$ scheme. This difference amounts to a three-loop (i.e., higher-order) effect in the calculation of the Higgs masses.

Electroweak interactions. The EW gauge sector of the NMSSM is determined by three fundamental parameters, i.e. the gauge coupling: ${ }^{10} g_{1}$ and $g_{2}$ and the combination of Higgs VEVs $v=\left(v_{u}^{2}+v_{d}^{2}\right)^{1 / 2}$. For a consistent determination of these three parameters, a choice of three physical inputs is required. The procedures adopted by the five codes differ in the choices of physical inputs, as well as, in the case of NMSSMCALC, the choice of renormalization scheme for the fundamental parameters.

1. FlexibleSUSY, SOFTSUSY and SPheno adapt again the approach of Ref. 110 to calculate $g_{1}$ and $g_{2}$. The three physical inputs are chosen as the $Z$ mass, the Fermi constant $G_{F}$ and the electromagnetic coupling of the SM at the scale $M_{Z}$ in the 5 -flavor scheme, $\alpha_{e m}^{(5), \overline{\mathrm{MS}}}\left(M_{Z}\right)$. The threshold corrections to $\alpha_{e m}$ from SUSY states, the charged Higgs and the top are calculated at the scale $M_{Z}$ as

$$
\begin{aligned}
& \alpha_{e m}^{\overline{\mathrm{DR}}}\left(M_{Z}\right)= \frac{\alpha_{e m}^{(5), \overline{\mathrm{MS}}}\left(M_{Z}\right)}{1-\Delta \alpha\left(M_{Z}\right)}, \\
& \Delta \alpha\left(M_{Z}\right)=\frac{\alpha}{2 \pi}\left(\frac{1}{3}-\frac{16}{9} \log \frac{m_{t}}{M_{Z}}-\frac{4}{9} \sum_{i=1}^{6} \log \frac{m_{\tilde{u}_{i}}}{M_{Z}}-\frac{1}{9} \sum_{i=1}^{6} \log \frac{m_{\tilde{d}_{i}}}{M_{Z}}\right. \\
&\left.\quad-\frac{4}{3} \sum_{i=1}^{2} \log \frac{m_{\tilde{\chi}_{i}^{+}}}{M_{Z}}-\frac{1}{3} \sum_{i=1}^{6} \log \frac{m_{\tilde{e}_{i}}}{M_{Z}}-\frac{1}{3} \log \frac{m_{H^{+}}}{M_{Z}}\right) .
\end{aligned}
$$

The Weinberg angle $\sin ^{\overline{\mathrm{DR}}} \Theta_{W}$ at the scale $M_{Z}$ is obtained iteratively from the above-computed $\alpha_{e m}^{\overline{\mathrm{DR}}}\left(M_{Z}\right)$, plus $G_{F}$ and $M_{Z}$, via the relation

$$
\left(\sin ^{\overline{\mathrm{DR}}} \Theta_{W} \cos ^{\overline{\mathrm{DR}}} \Theta_{W}\right)^{2}=\frac{\pi \alpha_{e m}^{\overline{\mathrm{DR}}}\left(M_{Z}\right)}{\sqrt{2} M_{Z}^{2} G_{F}\left(1-\delta_{r}\right)},
$$

${ }^{10}$ For the $U(1)$ coupling $g_{1}$ we adopt the SM normalization, i.e. $g_{1}=g^{\prime}$. 
where

$$
\begin{aligned}
& \delta_{r}=\hat{\rho} \frac{\Pi_{W W}^{T}(0)}{M_{W}^{2}}-\frac{\Re \mathrm{e} \Pi_{Z Z}^{T}\left(M_{Z}^{2}\right)}{M_{Z}^{2}}+\delta_{\mathrm{VB}}+\delta_{r}^{(2)} \\
& \hat{\rho}=\frac{1}{1-\Delta \hat{\rho}}, \quad \Delta \hat{\rho}=\Re \mathrm{e}\left[\frac{\Pi_{Z Z}^{T}\left(M_{Z}^{2}\right)}{\hat{\rho} M_{Z}^{2}}-\frac{\Pi_{W W}^{T}\left(M_{W}^{2}\right)}{M_{W}^{2}}\right]+\Delta \hat{\rho}^{(2)},
\end{aligned}
$$

$\Pi_{V V}^{T}\left(p^{2}\right) \quad(V=Z, W)$ are the $\overline{\mathrm{DR}}$-renormalized transverse parts of the self-energies of the vector bosons, computed at the renormalization scale $Q=M_{Z}$, and $\delta_{r}^{(2)}$ and $\Delta \hat{\rho}^{(2)}$ are two-loop corrections given in [114, 110]. The one-loop vertex and box corrections $\delta_{\mathrm{VB}}$ implemented into FlexibleSUSY and SOFTSUSY are from refs. [115, 116, 117, generalized to give the complete one-loop result for the NMSSM, while the ones used in SPheno are automatically generated with SARAH for the NMSSM. We remark that, in this approach, the physical value of the $W$ mass is an output of the calculation. At a generic scale $Q$, the relation between the pole mass $M_{V}$ of a gauge boson $V$ and its corresponding $\overline{\mathrm{DR}}$ mass reads

$$
M_{V}^{\overline{\mathrm{DR}}}(Q)=\sqrt{M_{V}^{2}+\Pi_{V V}^{T}\left(M_{V}^{2}\right)},
$$

where the self-energy is also computed at the scale $Q$.

In FlexibleSUSY $\sin ^{\overline{\mathrm{DR}}} \Theta_{W}$ can be alternatively calculated from the $\overline{\mathrm{DR}} W$ and $Z$ masses, via

$$
\sin ^{\overline{\mathrm{DR}}} \Theta_{W}\left(M_{Z}\right)=\sqrt{1-\left(\frac{M_{W}^{\overline{\mathrm{DR}}}\left(M_{Z}\right)}{M_{Z}^{\overline{\mathrm{DR}}}\left(M_{Z}\right)}\right)^{2}} .
$$

In this case the three physical inputs used to calculate $g_{1}$ and $g_{2}$ are $M_{Z}, M_{W}$ and $\alpha_{e m}^{(5), \overline{\mathrm{MS}}}\left(M_{Z}\right)$. This approach is the original one implemented in FlexibleSUSY 1.0.0 and when we refer to "out-of-the-box" results for FlexibleSUSY it will be using this option.

The so-obtained values for $\alpha_{e m}^{\overline{\mathrm{DR}}}$ and $\sin ^{\overline{\mathrm{DR}}} \Theta_{W}$ are used to get $g_{1}^{\overline{\mathrm{DR}}}\left(M_{Z}\right)$ and $g_{2}^{\overline{\mathrm{DR}}}\left(M_{Z}\right)$, which are then evolved to the SUSY scale using two-loop RGEs of the NMSSM.

2. NMSSMTools uses $M_{Z}, M_{W}$ and $G_{F}$ as physical inputs for the EW sector. It calculates the $\overline{\mathrm{DR}}$ values of $g_{1}$ and $g_{2}$ at the scale $M_{S U S Y}$ by making use of the relations between the fundamental EW parameters and the $\overline{\mathrm{DR}}$ masses of the gauge bosons, the latter computed at the scale $Q=$ $M_{S U S Y}$ as in eq. 26):

$$
\begin{aligned}
\sqrt{g_{1}^{\overline{\mathrm{DR}}}\left(M_{S U S Y}\right)^{2}+g_{2}^{\overline{\mathrm{DR}}}\left(M_{S U S Y}\right)^{2}} & =2 M_{Z}^{\overline{\mathrm{DR}}}\left(M_{S U S Y}\right) / v^{\overline{\mathrm{DR}}}\left(M_{S U S Y}\right), \\
g_{2}^{\overline{\mathrm{DR}}}\left(M_{S U S Y}\right) & =2 M_{W}^{\overline{\mathrm{DR}}}\left(M_{S U S Y}\right) / v^{\overline{\mathrm{DR}}}\left(M_{S U S Y}\right),
\end{aligned}
$$

with $v^{\overline{\mathrm{DR}}}\left(M_{S U S Y}\right)$ calculated via eq. 34$]^{11}$ below.

3. NMSSMCALC uses $M_{Z}, M_{W}$ and the electromagnetic gauge coupling $e$ as physical inputs for the EW sector. Moreover, it does not compute $\overline{\mathrm{DR}}$ values for the EW gauge couplings, but adopts the OS conditions

$$
g_{1}=e \frac{M_{Z}}{M_{W}}, \quad g_{2}=\frac{e}{\sqrt{1-M_{W}^{2} / M_{Z}^{2}}},
$$

where $M_{W}, M_{Z}$ and $e$ are the measured values.

\footnotetext{
${ }^{11}$ All expressions assume $v \simeq 246 \mathrm{GeV}$.
} 
Electroweak $V E V$. The last SM parameter that enters the calculation of the Higgs masses is $v$, which gets translated into the two Higgs VEVs $v_{d}$ and $v_{u}$ using the input value of $\tan \beta$. The parameter $v$ at the scale $M_{S U S Y}$ is obtained as follows in the different codes:

1. FlexibleSUSY and SOFTSUSY calculate the $\overline{\mathrm{DR}}$ value at $M_{Z}$ as

$$
v^{\overline{\mathrm{DR}}}\left(M_{Z}\right)=\frac{2 M_{Z}^{\overline{\mathrm{DR}}}\left(M_{Z}\right)}{\sqrt{g_{1}^{\overline{\mathrm{DR}}}\left(M_{Z}\right)^{2}+g_{2}^{\overline{\mathrm{DR}}}\left(M_{Z}\right)^{2}}} .
$$

$v^{\overline{\mathrm{DR}}}\left(M_{S U S Y}\right)$ is then obtained using two-loop RGEs with the $\xi$-dependent terms calculated in the Feynman gauge [83, 84].

2. SPheno calculates $v^{\overline{\mathrm{DR}}}$ directly at $M_{S U S Y}$ as

$$
v^{\overline{\mathrm{DR}}}\left(M_{S U S Y}\right)=\frac{2 M_{Z}^{\overline{\mathrm{DR}}}\left(M_{S U S Y}\right)}{\sqrt{g_{1}^{\overline{\mathrm{DR}}}\left(M_{S U S Y}\right)^{2}+g_{2}^{\overline{\mathrm{DR}}}\left(M_{S U S Y}\right)^{2}}},
$$

For completeness, we note that $v^{\overline{\mathrm{DR}}}\left(M_{Z}\right)$, which is used in the threshold corrections to the gauge and Yukawa couplings, is calculated separately via the relation

$$
v^{\overline{\mathrm{DR}}}\left(M_{Z}\right)=\sqrt{M_{Z}^{\overline{\mathrm{DR}}}\left(M_{Z}\right)^{2} \frac{\left(1-\sin ^{2} \Theta_{W}^{\overline{\mathrm{DR}}}\right) \sin ^{2} \Theta_{W}^{\overline{\mathrm{DR}}}}{\pi \alpha^{\overline{\mathrm{DR}}}}} .
$$

In this way, $v^{\overline{\mathrm{DR}}}\left(M_{Z}\right)$ and $v^{\overline{\mathrm{DR}}}\left(M_{S U S Y}\right)$ are not strictly correlated via an RGE evolution, but differences of a higher order are present.

3. NMSSMTools calculates $v^{\overline{\mathrm{DR}}}\left(M_{S U S Y}\right)$ via

$$
v^{\overline{\mathrm{DR}}}\left(M_{S U S Y}\right)^{-2}=\sqrt{2} G_{\mu}\left(1-\frac{\Pi_{W W}^{T}(0)}{M_{W}^{2}}-\delta_{\mathrm{VB}}\right),
$$

where $\delta_{\mathrm{VB}}$ denotes the one-loop correction to the muon decay amplitude. In the latter, NMSSMTools includes only the SM contribution.

4. NMSSMCALC uses also for $v$ an OS condition in terms of the three physical inputs:

$$
v=\frac{2 M_{W}}{e} \sqrt{1-M_{W}^{2} / M_{Z}^{2}}
$$

\subsubsection{Scales}

We have so far discussed differences in the threshold calculations, and how the codes perform those calculations at different scales. There are two other important scales involved in the calculation of the Higgs-boson masses: the scale at which the input parameters are taken by the codes, and the scale at which the pole masses are calculated. Thus, for a detailed comparison it is necessary that these scales be exactly the same in all codes. However, as mentioned in sec. 3.3, it is not possible to unify these two scales among the codes without modifications in NMSSMTools. In the following, we describe how these two scales are treated in general by the different codes, and how - and to what extent - they can be fixed to particular numerical values using the provided options in the input.

1. SPheno: If no scale is defined in the input, the input parameters are taken at $Q=\sqrt{m_{\tilde{t}_{1}} m_{\tilde{t}_{2}}}$ and the masses are calculated at the same scale. If a scale is set via the entries EXTPAR [0] or MODSEL [12], the input parameters are defined at this scale and the masses are calculated there as well.

2. NMSSMCALC uses a similar approach to SPheno and always calculates the masses at the same scale where the input parameters are taken. However, if no explicit scale is defined, the default choice is $Q=\sqrt{m_{\tilde{t}_{L}} m_{\tilde{t}_{R}}}$. 
3. SOFTSUSY by default takes the input scale to be the gauge-coupling unification scale. However, one may instead choose the input scale by either explicitly specifying it in EXTPAR [0] or by setting EXTPAR [0] to -1 in order to set the input scale to $Q=\sqrt{m_{\tilde{t}_{1}} m_{\tilde{t}_{2}}}$. In contrast to SPheno, changing this entry does not affect the scale at which the pole masses are calculated. SOFTSUSY calculates the pole masses at $Q=\sqrt{m_{\tilde{t}_{1}} m_{\tilde{t}_{2}}}$ by default, like SPheno. To change this one may set an alternative scale ${ }^{12}$ in the special SOFTSUSY block, using entry 4.

4. FlexibleSUSY: Both the parameter input scale and the scale at which the pole masses are calculated may be chosen by the user in the model file used to generate the code. In the NMSSM model file used in this section, both scales are set via the entry EXTPAR [0]. In the model file used in sec. 5 to study GUT scenarios the parameter input scale is set to be the gauge-coupling unification scale, and the scale where the pole masses are calculated is set to a generalization of the SUSY scale which reduces to $Q=\sqrt{m_{\tilde{t}_{1}} m_{\tilde{t}_{2}}}$ in the absence of family mixing.

5. NMSSMTools takes by default $Q=\sqrt{\left(2 m_{\tilde{u}_{L}}^{2}+m_{\tilde{u}_{R}}^{2}+m_{\tilde{d}_{R}}^{2}\right)} / 2$ for the input parameters and $Q^{\prime}=$ $\sqrt{m_{\tilde{t}_{L}} m_{\tilde{t}_{R}}}$ to calculate the Higgs, stop and sbottom pole masses. It is possible to change $Q$ to a fixed value via EXTPAR [0] or MINPAR [0], but fixing $Q^{\prime}$ is only possible by modifying the code (which is done for the present study). The running between the two scales is performed with two-loop RGEs for the NMSSM.

\subsubsection{Included two-loop corrections}

While all codes use complete calculations of the one-loop corrections to the Higgs masses 36, 37, 38, 39, the partial two-loop corrections implemented in the codes cover different contributions. All the mentioned two-loop corrections are calculated for zero external momentum.

1. NMSSMCALC includes only the two-loop corrections of $\mathcal{O}\left(\alpha_{s} \alpha_{t}\right)$ [1], but accounts also for the two-loop corrections to the electroweak VEV, which are omitted in the other codes.

2. SOFTSUSY, FlexibleSUSY and NMSSMTools use the two-loop corrections of $\mathcal{O}\left(\alpha_{s}\left(\alpha_{t}+\alpha_{b}\right)\right)$ which were calculated in the context of the NMSSM in Ref. 36. In addition, these codes use the MSSM results for the two-loop corrections of $\mathcal{O}\left(\left(\alpha_{t}+\alpha_{b}\right)^{2}\right)$ [69]. Moreover, SOFTSUSY and FlexibleSUSY include also routines to calculate the MSSM results for the tiny two-loop corrections involving $\alpha_{\tau}$ 67, 68, 69, 57].

3. SPheno performs a two-loop calculation in the gaugeless limit. Namely, any two-loop correction in the neutral Higgs sector that is independent of $g_{1}$ and $g_{2}$ is included. However, SPheno includes also the available routines for the NMSSM corrections of $\mathcal{O}\left(\alpha_{s}\left(\alpha_{t}+\alpha_{b}\right)\right)$, as well as MSSM corrections involving only Yukawa couplings similar to SOFTSUSY and FlexibleSUSY. If wished by the user, these routines can be used instead of the full calculation.

\subsection{Adjusting the codes}

To check if the differences between the codes listed in the previous section can really explain the sizable discrepancies in the Higgs-mass predictions, we have modified the codes, choosing SOFTSUSY as reference. This choice resulted from mere convenience: FlexibleSUSY is very close to SOFTSUSY, and the differences between the two codes were already fully understood. This made it easy to adapt FlexibleSUSY and left just three codes which required more modifications. In particular, we have changed the following:

- Calculation of the $\overline{\mathbf{D R}}$ parameters: Changing the codes to mimic the calculation of the running couplings of SOFTSUSY would be, of course, a big task beyond the scope of this comparison 13 Therefore, the chosen approach was to read in directly the values of $g_{i}, Y_{i}, v$ and $\tan \beta$ at the scale $Q$ as calculated by SOFTSUSY and use them in the calculation of the Higgs masses. This

\footnotetext{
${ }^{12}$ For this scale to be used directly it must be larger than $M_{Z}$. See documentation for full details.

${ }^{13}$ We stress that, in the context of this comparison, we double-checked the corresponding calculations performed by the codes and did not find any bugs.
} 


\begin{tabular}{|c|c|c|c|c|c|c|c|}
\hline & \multicolumn{2}{|c|}{ original } & \multicolumn{5}{|c|}{ modified } \\
\hline & one-loop & two-loop & one-loop & $\alpha_{s} \alpha_{t}$ & $\alpha_{s}\left(\alpha_{t}+\alpha_{b}\right)$ & $\begin{array}{c}\alpha_{s}\left(\alpha_{t}+\alpha_{b}\right) \\
+\operatorname{MSSM}\end{array}$ & 'full' \\
\hline \multicolumn{8}{|c|}{ TP1 } \\
\hline FlexibleSUSY & 119.48 & 123.55 & 119.73 & 124.82 & 124.82 & 123.81 & - \\
\hline NMSSMCALC & 115.49 & 120.34 & 119.75 & 124.85 & - & - & - \\
\hline NMSSMTOOLS & - & 123.52 & 119.75 & 124.84 & 124.84 & 123.84 & - \\
\hline SOFTSUSY & 119.75 & 123.84 & 119.75 & 124.84 & 124.84 & 123.84 & - \\
\hline SPHENO & 120.69 & 124.84 & 119.75 & 124.84 & 124.84 & 123.84 & 123.84 \\
\hline \multicolumn{8}{|c|}{ TP2 } \\
\hline FlexibleSUSY & 116.28 & 122.84 & 116.46 & 123.79 & 123.79 & 123.06 & - \\
\hline NMSSMCALC & 111.80 & 118.57 & 116.49 & 123.82 & - & - & - \\
\hline NMSSMTOOLS & - & 121.83 & 116.49 & 123.82 & 123.82 & 123.08 & - \\
\hline SOFTSUSY & 116.49 & 123.08 & 116.49 & 123.82 & 123.82 & 123.08 & - \\
\hline SPHENO & 118.00 & 124.74 & 116.49 & 123.81 & 123.81 & 123.08 & 123.05 \\
\hline \multicolumn{8}{|c|}{ TP3 } \\
\hline FlexibleSUSY & 124.16 & 126.58 & 124.15 & 127.55 & 127.55 & 126.59 & - \\
\hline NMSSMCALC & 121.76 & 124.86 & 124.15 & 127.56 & - & - & - \\
\hline NMSSMTOOLS & - & 127.28 & 124.15 & 127.56 & 127.56 & 126.60 & - \\
\hline SOFTSUSY & 124.15 & 126.59 & 124.15 & 127.56 & 127.56 & 126.59 & - \\
\hline SPHENO & 124.79 & 126.77 & 124.15 & 127.55 & 127.55 & 126.59 & 126.10 \\
\hline \multicolumn{8}{|c|}{ TP4 } \\
\hline FlexibleSUSY & 124.72 & 127.62 & 124.59 & 128.23 & 128.23 & 127.51 & - \\
\hline NMSSMCALC & 122.85 & 126.37 & 124.59 & 128.23 & - & - & - \\
\hline NMSSMTOOLS & - & 127.30 & 124.59 & 128.24 & 128.24 & 127.52 & - \\
\hline SOFTSUSY & 124.59 & 127.52 & 124.59 & 128.24 & 128.24 & 127.52 & - \\
\hline SPHENO & 124.89 & 126.62 & 124.59 & 128.23 & 128.23 & 127.52 & 126.33 \\
\hline \multicolumn{8}{|c|}{ TP5 } \\
\hline FlexibleSUSY & 122.53 & 125.08 & 122.54 & 126.11 & 126.11 & 125.11 & - \\
\hline NMSSMCALC & 121.95 & 123.14 & 122.54 & 126.12 & - & - & - \\
\hline NMSSMTOOLS & - & 126.95 & 122.54 & 126.12 & 126.12 & 125.12 & - \\
\hline SOFTSUSY & 122.54 & 125.12 & 122.54 & 126.12 & 126.12 & 125.12 & - \\
\hline SPHENO & 122.90 & 125.61 & 122.53 & 126.12 & 126.12 & 125.12 & 124.85 \\
\hline \multicolumn{8}{|c|}{ TP6 } \\
\hline FlexibleSUSY & 121.92 & 126.46 & 122.09 & 127.7 & 127.7 & 126.64 & - \\
\hline NMSSMCALC & 118.55 & 123.45 & 122.12 & 127.73 & - & - & - \\
\hline NMSSMTOOLS & - & 126.63 & 122.12 & 127.73 & 127.73 & 126.67 & - \\
\hline SOFTSUSY & 122.12 & 126.67 & 122.12 & 127.73 & 127.73 & 126.67 & - \\
\hline SPHENO & 123.37 & 131.29 & 122.09 & 127.71 & 127.71 & 126.65 & 129.91 \\
\hline
\end{tabular}

Table 6: The mass in GeV of the SM-like scalar after applying the modifications listed in sec. 4.2 for TP1-TP6 at the different loop levels and including different two-loop corrections. All two-loop corrections are for zero external momentum, while at one-loop the entire $p^{2}$ dependence is taken into account. "full" refers to a calculation which includes all corrections in the gaugeless limit where electroweak gauge couplings are neglected. Since the original version of NMSSMTools does not provide a flag to turn off the two-loop corrections, we don't give any one-loop value here. 
was done for NMSSMTools, NMSSMCALC and SPheno. In FlexibleSUSY no actual modifications to the code are made, but we will show results for both options for calculating the Weinberg angle. The option which matches the approach in SOFTSUSY will be treated as the modified version, while the approach implemented in FlexibleSUSY 1.0.0 will be treated as the original version.

- Renormalization scale: NMSSMTools was forced to calculate the Higgs mass at the same scale as all other codes.

- Renormalization scheme: The finite parts of the counterterms of the $W$ boson mass $\delta M_{W}$, the $Z$ boson mass $\delta M_{Z}$, and the electric charge $\delta e$ were put to zero in NMSSMCALC. Note that at two-loop level it is more convenient to introduce a counterterm for $v$, since in the gaugeless limit $\delta M_{Z}^{2}$ and $\delta M_{W}^{2}$ are zero, however, $\delta M_{Z}^{2} / M_{Z}^{2}$ and $\delta M_{W}^{2} / M_{W}^{2}$ not. The remaining combinations can be expressed by $\delta v$. This counterterm also needs to be set to zero to adjust to the other codes.

- Loop corrections: Of course no additional loop corrections have been implemented in any of the codes in the context of this analysis. However, we added options to the different codes to turn off specific loop corrections, to get a clear picture of the masses at one-loop and at two-loop including (i) $\mathcal{O}\left(\alpha_{s} \alpha_{t}\right)$, (ii) $\mathcal{O}\left(\alpha_{s}\left(\alpha_{t}+\alpha_{b}\right)\right)$ (iii) $\mathcal{O}\left(\alpha_{s}\left(\alpha_{t}+\alpha_{b}\right)\right)$ plus MSSM approximations for the corrections involving only Yukawa couplings, (iv) the full NMSSM calculation in the gaugeless limit.

\subsection{Masses after the adjustments}

The results for the SM-like Higgs mass after applying all changes to the codes are listed in Tab. 6 . Here, we disentangled also the different loop corrections at the one- and two-loop level. The entries $\left|Z_{i 1}^{H}\right|$ and $\left|Z_{i 3}^{H}\right|$ of the corresponding rotation matrix are listed in Tab. 7

The big differences that were observed before are totally gone when comparing equivalent calculations. Thus, for TP1-TP5 all codes show an impressive agreement at $\mathcal{O}\left(\alpha_{s} \alpha_{t}\right)$. The corrections of $\mathcal{O}\left(\alpha_{s} \alpha_{b}\right)$ are completely negligible for all considered points (they could, however, give a sizable effect for very large $\tan \beta)$. When including the purely Yukawa corrections at the two-loop level in the MSSM approximation, which is possible in all codes but NMSSMCALC, very good agreement is also found among the different tools. However, this approximation might not always be very good, and fails in particular for large $\lambda$. This is manifest in the comparison with the full NMSSM result, which is available only for SPheno.

\subsection{Size of the different effects}

We discuss now in turn the impact of the different changes we applied.

1. FlexibleSUSY: The entire difference between FlexibleSUSY and SOFTSUSY comes from the calculation of the Weinberg angle $\sin ^{\overline{\mathrm{DR}}} \Theta_{W}$ at the scale $M_{Z}$, as described in Section 4.1.2.

2. SPheno: In Tab. 8 the effects of adjusting successively the Yukawa couplings, the gauge couplings, and the electroweak VEV are shown. One can see that the biggest change comes from the Yukawa couplings. Of course, the top Yukawa coupling plays the main role.

3. NMSSMCALC: Tab. 9 shows the effects of applying successively the different adjustments. Here we show the effect of changing the Yukawa couplings, changing the renormalization of the EW sector, and neglecting $\mathcal{O}\left(\alpha_{s} \alpha_{t}\right)$ contributions due to the conversion of the electroweak VEV to the $\overline{\mathrm{DR}}$ parameter, which were omitted in the other spectrum calculators. The effect of the latter on the Higgs masses is proportional to $\lambda$, and hence more visible for large $\lambda$.

4. NMSSMTools: Tab. 10 shows the effects of applying successively the different adjustments: the effects of adjusting successively the scale $Q^{\prime}$ for the Higgs-mass calculation, the running Yukawa and gauge couplings and the running electroweak VEV. The changes in the scale have only an effect for TP2, because for the other points $Q$ was chosen as $\sqrt{m_{\tilde{t}_{L}} m_{\tilde{t}_{R}}}$, which coincides with the scale $Q^{\prime}$ used by NMSSMTools. The only sizable effects are due to the top Yukawa coupling and the changes in the VEV. 


\begin{tabular}{|c|c|c|c|c|}
\hline & \multicolumn{2}{|c|}{ original } & \multicolumn{2}{|l|}{ modified } \\
\hline & one-loop & two-loop & $\begin{array}{c}\alpha_{s}\left(\alpha_{t}+\alpha_{b}\right) \\
+\mathrm{MSSM}\end{array}$ & 'full' \\
\hline \multicolumn{5}{|c|}{ TP1 } \\
\hline FlexibleSUSY & $0.1039,0.0076$ & $0.1039,0.0076$ & $0.1039,0.00760 .1039,0.00760 .1039,0.0076$ & - \\
\hline NMSSMCALC & $0.1039,0.0076$ & $0.1039,0.0076$ & $0.1039,0.00760 .1039,0.0076$ & - \\
\hline NMSSMTOOLS & - & $0.1039,0.0076$ & $0.1039,0.00760 .1039,0.00760 .1039,0.0076$ & - \\
\hline SOFTSUSY & $0.1039,0.0076$ & $0.1039,0.0076$ & $0.1039,0.00760 .1039,0.00760 .1039,0.0076$ & - \\
\hline SPHENO & $0.1040,0.0076$ & $0.1040,0.0074$ & $\begin{array}{lllll}0.1039,0.0076 & 0.1039,0.0076 & 0.1039,0.0076 & 0\end{array}$ & $039,0.0075$ \\
\hline \multicolumn{5}{|c|}{ TP2 } \\
\hline FlexibleSUSY & $0.1034,0.0004$ & $0.1034,0.0004$ & $0.1034,0.00040 .1034,0.00040 .1034,0.0004$ & - \\
\hline NMSSMCALC & $0.1034,0.0004$ & $0.1034,0.0004$ & $0.1034,0.00040 .1034,0.0004$ & - \\
\hline NMSSMTOOLS & - & $0.1038,0.0004$ & $0.1034,0.00040 .1034,0.00040 .1034,0.0004$ & - \\
\hline SOFTSUSY & $0.1034,0.0004$ & $0.1034,0.0004$ & $0.1034,0.00040 .1034,0.00040 .1034,0.0004$ & - \\
\hline SPHENO & $0.1035,0.0004$ & $0.1035,0.0004$ & $0.1034,0.00040 .1034,0.00040 .1034,0.00040$ & $034,0.0004$ \\
\hline \multicolumn{5}{|c|}{ TP3 } \\
\hline FlexibleSUSY & $0.2687,0.2975$ & $0.2813,0.2470$ & $0.2693,0.29700 .2835,0.2400 \quad 0.2819,0.2465$ & - \\
\hline NMSSMCALC & $0.2664,0.3081$ & $0.2811,0.2502$ & $0.2705,0.29350 .2842,0.2385$ & - \\
\hline NMSSMTOOLS & - & $0.2782,0.2708$ & $0.2705,0.2935 \quad 0.2842,0.2384 \quad 0.2828,0.2442$ & - \\
\hline SOFTSUSY & $0.2693,0.2970$ & $0.2820,0.2460$ & $0.2693,0.29700 .2836,0.23990 .2820,0.2460$ & - \\
\hline SPHENO & $0.2701,0.2951$ & $0.2725,0.2851$ & $0.2693,0.29690 .2836,0.2398 \quad 0.2820,0.24590$ & $719,0.2863$ \\
\hline \multicolumn{5}{|c|}{ TP4 } \\
\hline FlexibleSUSY & $0.4852,0.3689$ & $0.4866,0.3712$ & $0.4853,0.36980 .4862,0.39380 .4868,0.3722$ & - \\
\hline NMSSMCALC & $0.4858,0.3625$ & $0.4869,0.3832$ & $0.4845,0.40980 .4836,0.4476$ & - \\
\hline NMSSMTOOLS & - & $0.4855,0.4501$ & $0.4845,0.40980 .4837,0.44720 .4851,0.4235$ & - \\
\hline SOFTSUSY & $0.4853,0.3700$ & $0.4867,0.3725$ & $\begin{array}{llll}0.4853,0.3700 & 0.4862,0.3941 & 0.4867,0.3725\end{array}$ & - \\
\hline SPHENO & $0.4852,0.3810$ & $0.4835,0.4262$ & $0.4853,0.3698 \quad 0.4862,0.39390 .4867,0.37230$ & $40,0.4133$ \\
\hline \multicolumn{5}{|c|}{ TP5 } \\
\hline FlexibleSUSY & $0.0171,0.8787$ & $0.2189,0.4681$ & $0.0205,0.87540 .2445,0.38810 .2191,0.4685$ & - \\
\hline NMSSMCALC & $0.0154,0.9162$ & $0.2055,0.5099$ & $0.1259,0.70200 .2621,0.3276$ & - \\
\hline NMSSMTOOLS & - & $0.1974,0.5396$ & $0.1259,0.70200 .2621,0.32760 .2485,0.3758$ & - \\
\hline SOFTSUSY & $0.0208,0.8749$ & $0.2195,0.4679$ & $0.0208,0.87490 .2447,0.38760 .2195,0.4679$ & - \\
\hline SPHENO & $0.0605,0.8192$ & $0.1958,0.5372$ & $0.0206,0.8752 \quad 0.2449,0.387 \quad 0.2197,0.46720$ & $306,0.5766$ \\
\hline \multicolumn{5}{|c|}{ TP6 } \\
\hline FlexibleSUSY & $0.2741,0.1946$ & $0.2741,0.1950$ & $0.2746,0.19370 .2747,0.19420 .2747,0.1941$ & - \\
\hline NMSSMCALC & $0.2742,0.1947$ & $0.2746,0.1947$ & $0.2748,0.19350 .2749,0.1939$ & - \\
\hline NMSSMTOOLS & - & $0.2743,0.1953$ & $0.2748,0.1935 \quad 0.2749,0.19390 .2749,0.1939$ & - \\
\hline SOFTSUSY & $0.2746,0.1937$ & $0.2747,0.1941$ & $0.2746,0.19370 .2747,0.19410 .2747,0.1941$ & - \\
\hline SPHENO & $0.2748,0.1938$ & $0.2712,0.2039$ & $0.2746,0.19370 .2747,0.19420 .2747,0.19410$ & $711,0.2037$ \\
\hline
\end{tabular}

Table 7: Absolute value of the $H_{d}$ and singlet components $\left(\left|Z_{i 1}^{H}\right|,\left|Z_{i 3}^{H}\right|\right)$ of the SM-like Higgs with index $i$ after the modifications listed in sec. 4.2 for TP1-TP6. Because of the size of the table, we do not show explicitly the largest entry $\left|Z_{i 2}^{H}\right|$, but it can easily be calculated as $\left|Z_{i 2}^{H}\right|^{2}=1-\left|Z_{i 1}^{H}\right|^{2}-\left|Z_{i 3}^{H}\right|^{2}$. The remaining differences are caused by the external momentum $p^{2}$ used to calculate $Z^{H}$ : NMSSMCALC and NMSSMTools set $p^{2}=0$, while the other codes set $p^{2}=m_{h_{1}}^{2}$. The conventions for the given corrections are the same as in Tab. 6. but we do not show the results for $\alpha_{s}\left(\alpha_{b}+\alpha_{t}\right)$ explicitly because they fully agree with the ones for $\alpha_{s} \alpha_{t}$ for the number of digits used here. 


\begin{tabular}{|c|c|ccc|c|}
\hline Point & original & $Y$ & $g$ & $v$ & modified \\
\hline TP1 & 124.84 & 123.65 & 123.61 & 123.84 & 123.84 \\
TP2 & 124.74 & 123.18 & 123.13 & 123.05 & 123.05 \\
TP3 & 126.77 & 126.06 & 126.00 & 126.10 & 126.10 \\
TP4 & 126.62 & 126.21 & 126.16 & 126.33 & 126.33 \\
TP5 & 125.61 & 124.89 & 124.84 & 124.85 & 124.85 \\
TP6 & 131.29 & 130.06 & 130.01 & 129.91 & 129.91 \\
\hline
\end{tabular}

Table 8: The Higgs prediction for the SM-like Higgs mass by SPheno after applying successively the different adjustments for the Yukawas $(Y)$, gauge couplings $(g)$, and the electroweak VEV $(v)$. Here, "original" refers to the results when using the code without any modification, while for "modified" all adjustments are turned on.

\begin{tabular}{|c|c|ccc|c|}
\hline Point & original & $Y$ & $\delta_{1}$ & $\delta_{2}$ & modified \\
\hline TP1 & 120.34 & 124.41 & 124.85 & 124.85 & 124.85 \\
TP2 & 118.57 & 123.31 & 123.82 & 123.82 & 123.82 \\
TP3 & 124.86 & 127.55 & 127.50 & 127.56 & 127.56 \\
TP4 & 126.37 & 128.32 & 128.18 & 128.23 & 128.23 \\
TP5 & 123.14 & 126.21 & 126.03 & 126.12 & 126.12 \\
TP6 & 123.45 & 127.26 & 127.55 & 127.73 & 127.73 \\
\hline
\end{tabular}

Table 9: The prediction for the SM-like Higgs mass by NMSSMCALC after adjusting the $\overline{\mathrm{DR}}$ Yukawa couplings $(Y)$; performing a $\overline{\mathrm{DR}}$ renormalization of the EW sector by changing the values of $g_{1}, g_{2}$ and $v$ and removing the finite parts of the one-loop counter-terms $\delta M_{W}, \delta M_{Z}$ and $\delta e\left(\delta_{1}\right)$; removing also the finite counterterm for $v$ at the two-loop level $\left(\delta_{2}\right)$. The same conventions as for Tab. 8 are used.

One can see from this comparison that the main source of discrepancies between the predictions of the five codes is the $\overline{\mathrm{DR}}$-renormalized top Yukawa coupling used in the calculation of the Higgs masses: differences in the determination of $Y_{t}^{\overline{\mathrm{DR}}}(Q)$ have a larger impact than additional two-loop corrections to the Higgs mass matrix. In particular, the fact that NMSSMCALC includes only (S)QCD corrections in the calculation of $Y_{t}^{\overline{\mathrm{DR}}}(Q)$ results in a prediction for the SM-like Higgs mass a few $\mathrm{GeV}$ below the ones of the other codes. The results in tables 6 and 9 suggest that the inclusion of additional one-loop corrections in the calculation of that coupling by NMSSMCALC would account for the bulk of the corresponding two-loop corrections to the Higgs masses, thus reducing the discrepancies with the other codes. However, the precise calculation of the Higgs decays in NMSSMCALC includes only (S)QCD corrections. Therefore, with the aim of preserving the overall consistency of the calculation, the authors of NMSSMCALC include only (S)QCD corrections in $Y_{t}^{\overline{\mathrm{DR}}}(Q)$ as well.

\begin{tabular}{|c|c|ccccc|}
\hline Point & original & $Q^{\prime}=Q$ & $Y$ & $g$ & $v$ & modified \\
\hline TP1 & 123.52 & 123.52 & 123.96 & 123.99 & 123.84 & 123.84 \\
TP2 & 121.83 & 121.44 & 123.46 & 123.52 & 123.08 & 123.08 \\
TP3 & 127.28 & 127.28 & 127.43 & 127.43 & 126.60 & 126.60 \\
TP4 & 127.30 & 127.30 & 127.13 & 127.07 & 127.52 & 127.52 \\
TP5 & 126.95 & 126.95 & 127.34 & 127.45 & 125.12 & 125.12 \\
TP6 & 126.63 & 126.63 & 127.56 & 127.66 & 126.67 & 126.67 \\
\hline
\end{tabular}

Table 10: Changes in the prediction by NMSSMTools for the SM-like Higgs mass after forcing the SUSY scale and the scale for the mass calculation to be identical $\left(Q^{\prime}=Q\right)$, changing the Yukawa couplings $(Y)$, the gauge couplings $(g)$, and the electroweak VEV $(v)$. The same conventions as for Tab. 8 are used. 


\section{Expected effects for GUT scenarios}

We have focused so far on the Higgs-mass calculation in which all SUSY parameters are fixed by the input at the SUSY scale. However, most codes offer also the possibility to study a GUT scenario where the soft SUSY-breaking parameters are fixed at the GUT scale. The differences among the codes discussed in the previous sections are going to play an important role here as well. Indeed, because of the additional RGE running, the discrepancies between the results of the different codes are usually of the same size as, and often even bigger than those found in the case of SUSY-scale inputs with comparable parameter choices. In particular, the differences in the determination of the $\overline{\mathrm{DR}}$ couplings can play a crucial role, because the different values for the couplings at the SUSY scale affect the entire running between $M_{S U S Y}$ and $M_{G U T}$. This is especially important for points in which the input value of $\lambda$ at $M_{S U S Y}$ is close to the perturbativity limit. Small changes in the threshold corrections to the weak-scale couplings can change the value of $\lambda$ at the GUT scale considerably. This will then change the running of all soft terms from the GUT scale to the SUSY scale. While the modifications in the sfermion parameters are not necessarily large, $A_{\lambda}$ and $A_{\kappa}$ can be strongly affected. This will then have an impact on the singlet-like scalar.

To demonstrate this effect, we consider the two points given in Tab. 11 . Both points provide an SM-like Higgs mass in the preferred range together with a light singlet, but the first (GTP1) is characterized by small values of $\lambda$ and $\kappa$, while the second (GTP2) is characterized by larger values of those couplings, not far below the perturbativity limit. To show the large sensitivity of GTP2 compared to GTP1 to the parameters at the SUSY scale, we vary the top mass within the uncertainty: $m_{t}=173.2 \pm 1.5 \mathrm{GeV}$. The masses of the three CP-even Higgs bosons computed by the four codes that allow for GUT-scale boundary conditions, i.e. FlexibleSUSY, NMSSMTools, SOFTSUSY and SPheno, are shown in Tab. 12. We have used for this comparison the un-tuned versions of all codes.

\begin{tabular}{|c|ccc|cccccc|}
\hline & $m_{0}$ & $M_{1 / 2}$ & $A_{0}$ & $\tan \beta$ & $\lambda$ & $\kappa$ & $A_{\lambda}$ & $A_{\kappa}$ & $\mu_{\text {eff }}$ \\
\hline GTP1 & 1000 & 1000 & -3000 & 10 & 0.05 & 0.05 & -150 & -300 & 100 \\
GTP2 & 1000 & 1000 & -3000 & 10 & 0.48 & 0.46 & -150 & -300 & 100 \\
\hline
\end{tabular}

Table 11: Input values for GTP1 and GTP2. All dimensionful parameters in units of GeV. The parameters in the left section of the table are defined at the GUT scale, whereas those in the right section are defined at the scale $M_{S U S Y}$, with the exception of $\tan \beta$ which is defined at $M_{Z}$.

\begin{tabular}{|c|ccc|}
\hline & $m_{t}=171.7 \mathrm{GeV}$ & $m_{t}=173.2 \mathrm{GeV}$ & $m_{t}=174.7 \mathrm{GeV}$ \\
\hline FlexibleSUSY & $100.1,122.2,951.9$ & $100.0,123.3,967.0$ & $100.0,124.5,982.0$ \\
NMSSMTools & $100.0,119.3,982.1$ & $100.0,120.1,974.4$ & $99.9,120.8,1014.0$ \\
SOFTSUSY & $100.1,122.5,958.6$ & $100.0,123.6,974.4$ & $100.0,124.8,990.2$ \\
SPheno & $100.0,123.7,976.6$ & $99.9,124.9,992.3$ & $99.9,126.0,1008.0$ \\
\hline \hline & $m_{t}=171.7 \mathrm{GeV}$ & $m_{t}=173.2 \mathrm{GeV}$ & $m_{t}=174.7 \mathrm{GeV}$ \\
\hline FlexibleSUSY & $102.8,123.3,961.9$ & $97.9,124.6,974.1$ & $92.5,126.1,986.1$ \\
NMSSMTools & $96.6,118.8,987.7$ & $91.4,119.1,1000.8$ & no output \\
SOFTSUSY & $102.4,123.7,968.2$ & $97.3,125.2,981.1$ & $91.4,126.7,994.0$ \\
SPheno & $95.1,125.3,981.9$ & $89.3,126.8,994.6$ & $82.5,128.3,1007.5$ \\
\hline
\end{tabular}

Table 12: The masses of the three CP-even Higgs bosons, $m_{h_{1,2}, 3}$, for GTP1 (top) and GTP2 (bottom) for three different values of the top quark mass, $m_{t}$.

We see that, for GTP1, the SM-like Higgs mass varies by about $2 \mathrm{GeV}$ for all codes when $m_{t}$ is varied within one standard deviation, while the light-singlet mass is fairly independent of the top mass, and the predictions of all codes for the light-singlet mass agree within $0.1 \mathrm{GeV}$. For GTP2, the variation with $m_{t}$ in the SM-like Higgs mass is about $3 \mathrm{GeV}$, but the singlet is very sensitive to the value of the top mass: its mass can change by about $10 \mathrm{GeV}$, and the different codes predict masses that differ from each other by several $\mathrm{GeV}$. In addition, for $m_{t}=174.7 \mathrm{GeV}$ NMSSMTools runs into the Landau pole and is not able to calculate the spectrum. The situation can be even more dramatic 
when taking for instance the benchmark points proposed in Ref. [118: for each of the three points BP1-BP3, at least two codes do not produce an output. When they are forced to produce an output (for instance by ignoring tachyonic states which appear at tree-level), the differences between the codes can reach $20-30 \mathrm{GeV}$ for the light singlet.

Points with very large $\lambda$ at the SUSY scale are attractive since they seem to soften the SUSY finetuning problem [11, 12, 13, 14, 15, 16, 17, 18, 19, 20]. However, if $\lambda$ is too close to the perturbativity limit, the output produced by the spectrum generators has to be taken with some care: these points can be very sensitive to small variations of the input parameters. Changing the SM input parameters within their respective uncertainties - or changing the details of the determination of the running couplings - can lead to a completely different spectrum, or even result in the spectrum generator classifying the parameter point as unphysical.

\section{Conclusions}

We have discussed the differences in the predictions for the scalar Higgs masses and mixings by public spectrum generators for the NMSSM. It has been shown that sizeable discrepancies can show up for various parameter points, but the origins of those discrepancies are fully understood. The main sources of discrepancies are in the approach used to calculate the running parameters that enter the Higgs-mass calculation. The most important parameter here is the top Yukawa coupling. Further discrepancies arise from the different accuracy of the two-loop corrections included in the codes.

This has important consequences for phenomenological studies: For MSSM-like points, FlexibleSUSY, NMSSMTools, SOFTSUSY and SPheno usually agree quite well with each other, while NMSSMCALC returns in general a smaller value for the SM-like Higgs boson mass. The main reasons for this discrepancy are the value of $Y_{t}^{\overline{\mathrm{DR}}}$ at $M_{S U S Y}$, which is always smaller in NMSSMCALC, and the inclusion of the MSSM results for the $\mathcal{O}\left(\left(\alpha_{t}+\alpha_{b}\right)^{2}\right)$ corrections in FlexibleSUSY, NMSSMTools and SOFTSUSY but not in NMSSMCALC. For MSSM-like points, the MSSM results provide a good approximation to the calculation implemented in SPheno which includes the NMSSM specific effects. On the other hand, this approximation usually fails for large values of $\lambda$ and/or if light singlet states are present. In these cases, the results of FlexibleSUSY, NMSSMTools and SOFTSUSY have to be taken with care because it might be that the MSSM approximation has even the wrong sign compared to the full NMSSM calculation [40. For these cases SPheno, with the additional NMSSM specific contributions, is expected to provide the most reliable prediction, with the caveat that if states in the loop become very light, the calculation can be plagued by intrinsic problems of the effective-potential calculation [119, 120, 121]. NMSSMCALC, on the other hand, is the only code that includes two-loop $\mathcal{O}\left(\alpha_{t} \alpha_{s}\right)$ corrections to $v$, which become non-negligible for large values of $\lambda$.

It has been demonstrated with one example, that the differences can be much more pronounced for GUT scenarios with values of $\lambda$ close to the perturbativity limit. The differences in the threshold calculations among the codes can cause huge discrepancies in particular for light singlets and the predicted masses can differ by $10 \mathrm{GeV}$ and more.

In general, comparing the predictions of different codes may provide a ballpark indication for the theoretical uncertainty of the Higgs mass calculation. However, even if these codes agree very well, one should not necessarily assume that the uncertainty is small: it is known from the MSSM that spectrum generators performing a $\overline{\mathrm{DR}}$ calculation (SOFTSUSY, SPheno, Suspect [122]) can agree quite well, while sizeable differences to the OS calculation of FeynHiggs exists. The differences are assumed to come from the missing electroweak corrections and momentum dependence at two-loop level as well as from the dominant three-loop corrections. These are the effects that underlie the often-quoted estimate of a $3 \mathrm{GeV}$ uncertainty for the SM-like Higgs mass in the MSSM [57, 105]. To get an estimate of the remaining theoretical uncertainty in the NMSSM, a comparison between different renormalization schemes will therefore be necessary. This is deferred to future work, when further publicly available codes will have implemented OS renormalization. However, even the validity of such an estimate is under discussion already in the MSSM, in particular for a heavier SUSY scale of $2 \mathrm{TeV}$ and larger. Calculations based on the effective field theory approach, which become valid in this mass range, 
usually predict a SM-like Higgs mass that is lower by a few $\mathrm{GeV}$ compared to the prediction from dedicated tools for the MSSM [123, 124, 125].

With this work an important first step has been taken to understand the large differences in the Higgs mass computations of the various public NMSSM codes. For the investigated codes these differences are now completely understood and it has been shown that discrepancies of more than $3 \mathrm{GeV}$ are not unusual taking into account the different corrections implemented. To further pin down the residual theoretical uncertainty the next natural step will be to extend this investigation to the comparison of different renormalization schemes.

\section{Acknowledgements}

We thank Peter Drechsel, Mark D. Goodsell, Werner Porod, Heidi Rzehak, Michael Spira, Kathrin Walz and Georg Weiglein for many useful discussions.

This work was supported in part by the Research Executive Agency (REA) of the European Commission under the Initial Training Networks "INVISIBLES" (PITN-GA-2011-289442) and "Higgs-Tools" (PITN-GA-2012-316704), and by the European Research Council (ERC) under the Advanced Grant "Higgs@LHC" (ERC-2012-ADG_20120216-321133). The work of P. S. at LPTHE is supported in part by French state funds managed by the ANR (ANR-11-IDEX-0004-02) in the context of the ILP LABEX (ANR-10-LABX-63). The work of P. A. was supported by the ARC Centre of Excellence for Particle Physics at the Terascale.

\section{Appendix A. Used options in input files}

Appendix A.1. FlexibleSUSY

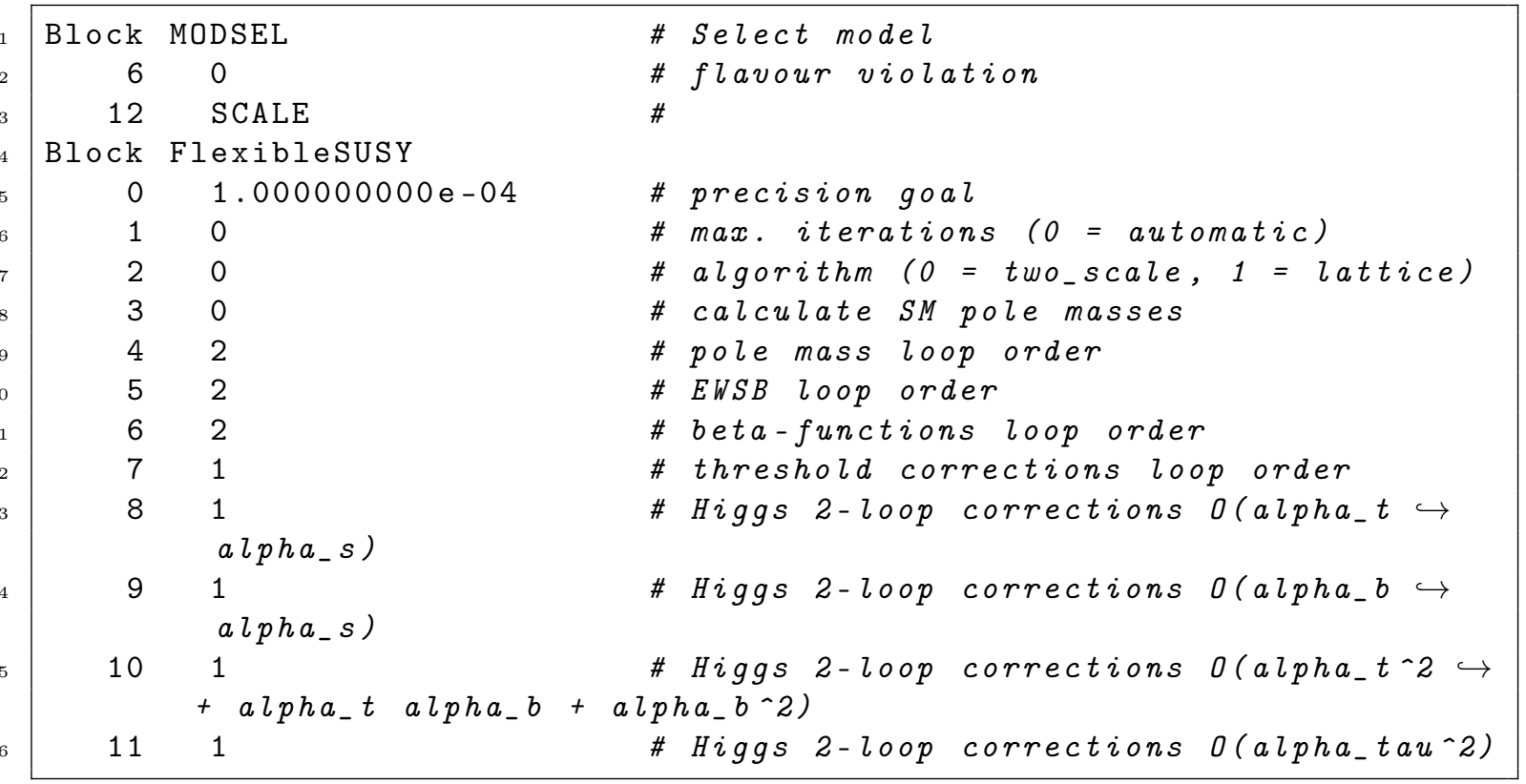

Appendix A.2. NMSSMCALC




Appendix A.3. NMSSMTools

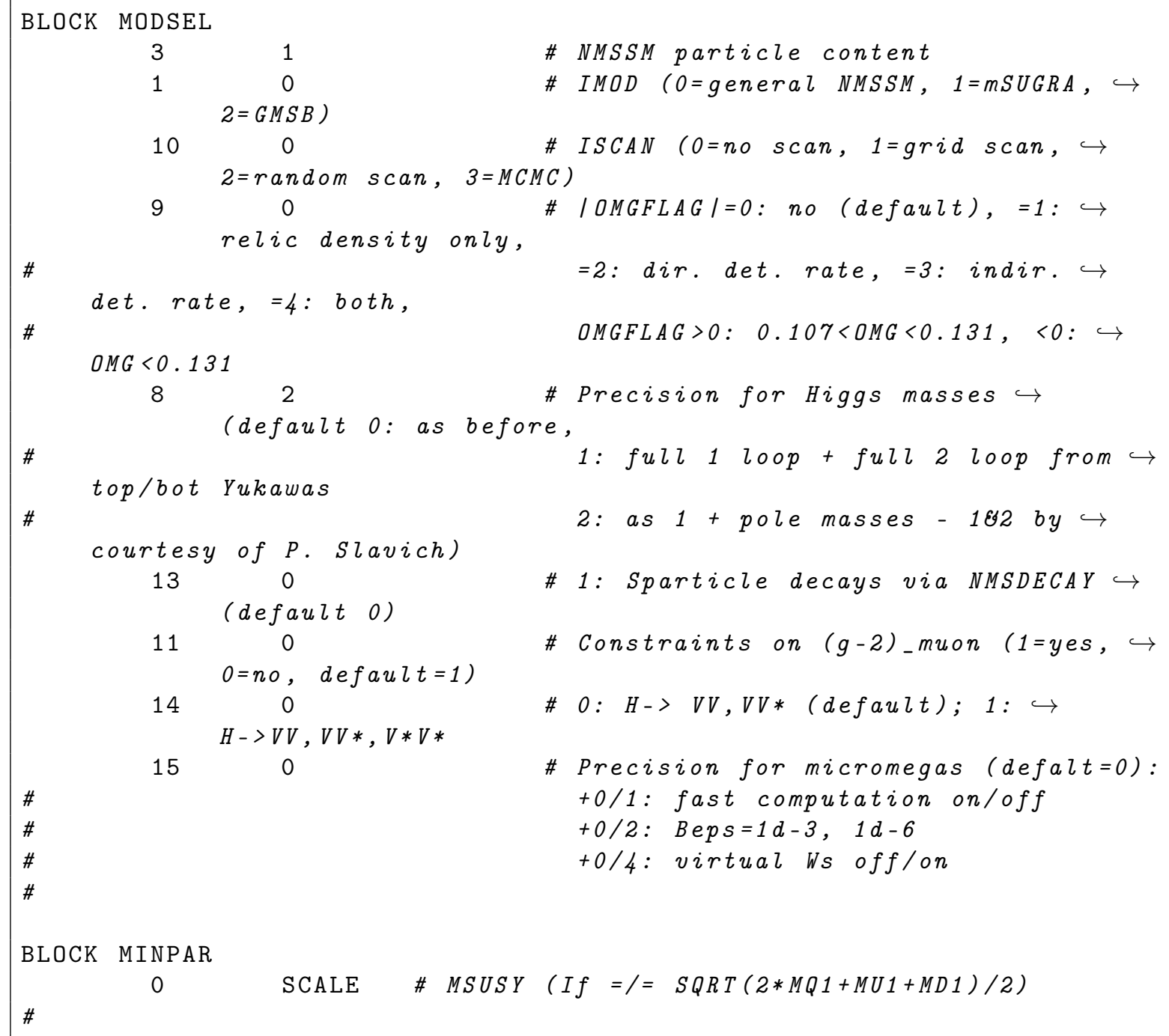

Appendix A.4. SOFTSUSY

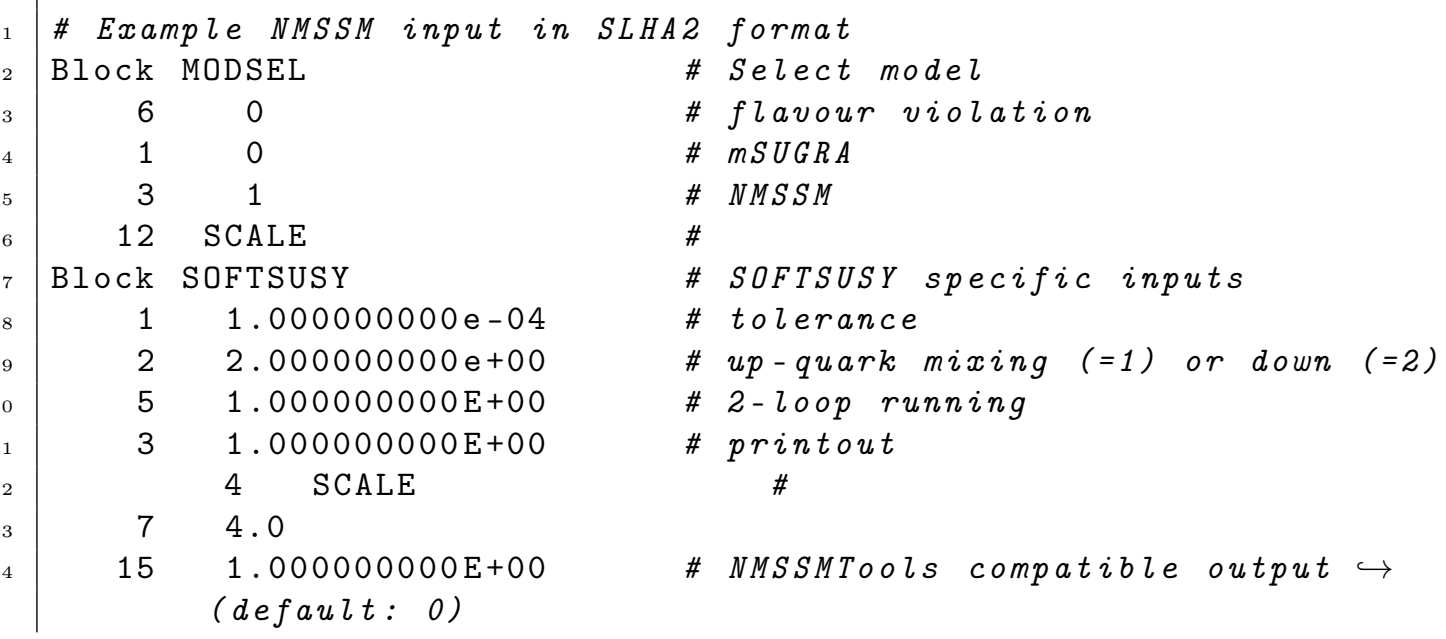




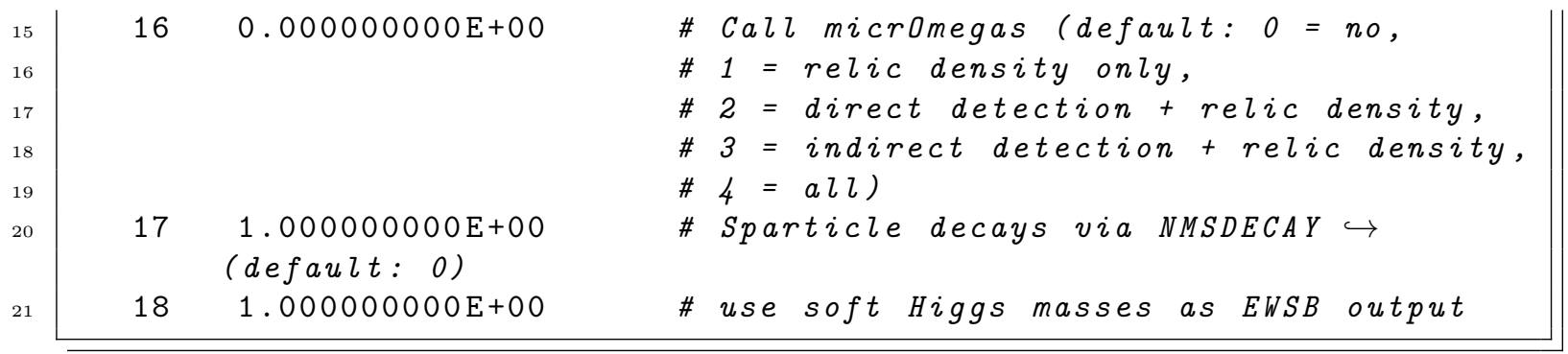

Appendix A.5. SPheno

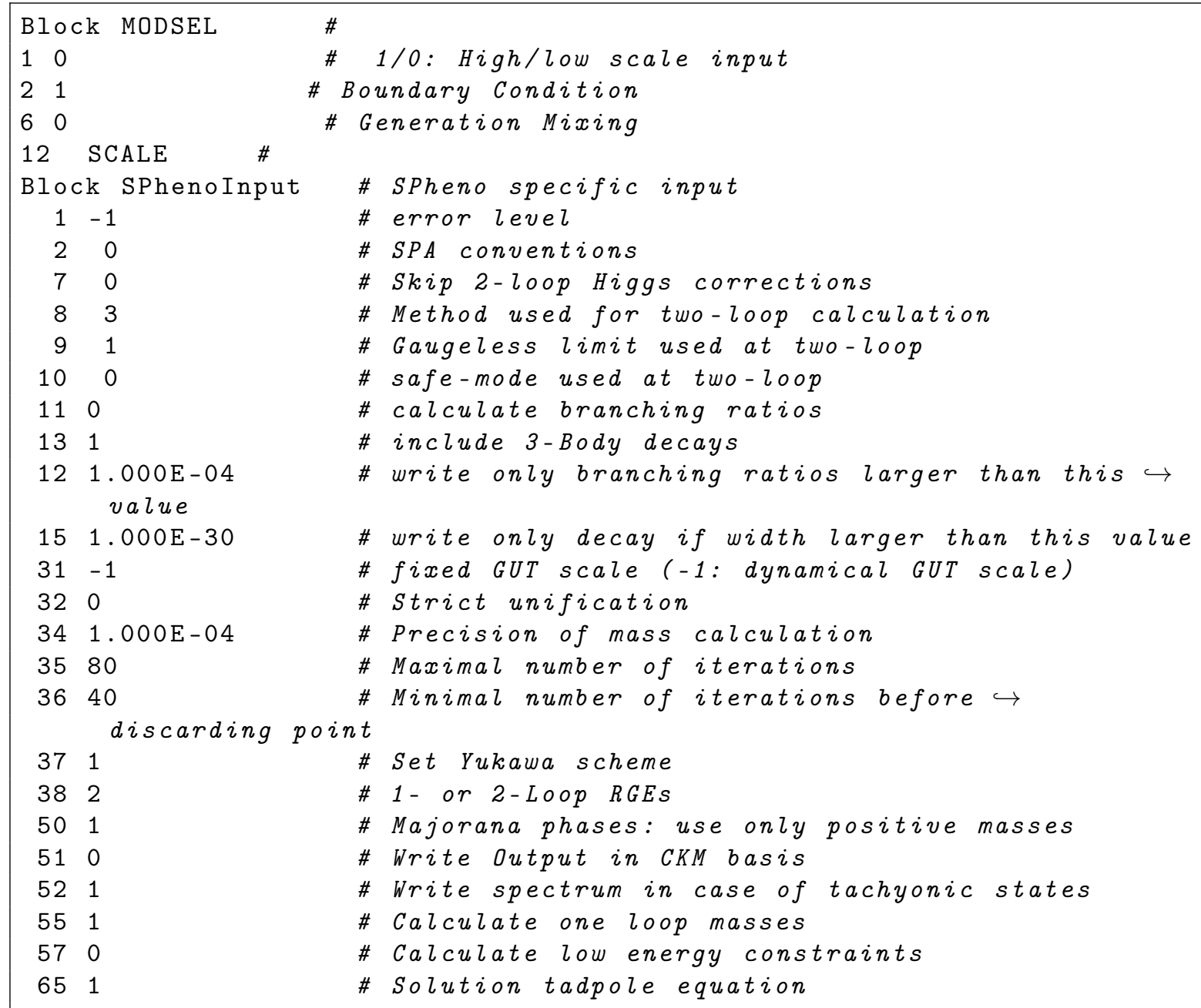

\section{References}

[1] S. Chatrchyan et al., "Observation of a new boson at a mass of $125 \mathrm{GeV}$ with the CMS experiment at the LHC," Phys.Lett., vol. B716, pp. 30-61, 2012, 1207.7235.

[2] G. Aad et al., "Observation of a new particle in the search for the Standard Model Higgs boson with the ATLAS detector at the LHC," Phys.Lett., vol. B716, pp. 1-29, 2012, 1207.7214.

[3] J. Camargo-Molina, B. O'Leary, W. Porod, and F. Staub, "Stability of the CMSSM against sfermion VEVs," JHEP, vol. 1312, p. 103, 2013, 1309.7212.

[4] N. Blinov and D. E. Morrissey, "Vacuum Stability and the MSSM Higgs Mass," JHEP, vol. 1403, p. 106, 2014, 1310.4174 . 
[5] D. Chowdhury, R. M. Godbole, K. A. Mohan, and S. K. Vempati, "Charge and Color Breaking Constraints in MSSM after the Higgs Discovery at LHC," JHEP, vol. 1402, p. 110, 2014, 1310.1932.

[6] J. Camargo-Molina, B. Garbrecht, B. O'Leary, W. Porod, and F. Staub, "Constraining the Natural MSSM through tunneling to color-breaking vacua at zero and non-zero temperature," Phys.Lett., vol. B737, pp. 156-161, 2014, 1405.7376 .

[7] U. Chattopadhyay and A. Dey, "Exploring MSSM for Charge and Color Breaking and Other Constraints in the Context of Higgs@125 GeV," JHEP, vol. 1411, p. 161, 2014, 1409.0611.

[8] M. Maniatis, "The Next-to-Minimal Supersymmetric extension of the Standard Model reviewed," Int. J. Mod. Phys., vol. A25, pp. 3505-3602, 2010, 0906.0777.

[9] U. Ellwanger, C. Hugonie, and A. M. Teixeira, "The Next-to-Minimal Supersymmetric Standard Model," Phys.Rept., vol. 496, pp. 1-77, 2010, 0910.1785

[10] U. Ellwanger and C. Hugonie, "The Upper bound on the lightest Higgs mass in the NMSSM revisited," Mod.Phys.Lett., vol. A22, pp. 1581-1590, 2007, hep-ph/0612133.

[11] M. Bastero-Gil, C. Hugonie, S. King, D. Roy, and S. Vempati, "Does LEP prefer the NMSSM?," Phys.Lett., vol. B489, pp. 359-366, 2000, hep-ph/0006198.

[12] R. Dermisek and J. F. Gunion, "Consistency of LEP event excesses with an h - > aa decay scenario and low-finetuning NMSSM models," Phys.Rev., vol. D73, p. 111701, 2006, hep-ph/0510322

[13] U. Ellwanger, G. Espitalier-Noel, and C. Hugonie, "Naturalness and Fine Tuning in the NMSSM: Implications of Early LHC Results," JHEP, vol. 1109, p. 105, 2011, 1107.2472.

[14] G. G. Ross and K. Schmidt-Hoberg, "The Fine-Tuning of the Generalised NMSSM," Nucl.Phys., vol. B862, pp. 710719, 2012, 1108.1284.

[15] G. G. Ross, K. Schmidt-Hoberg, and F. Staub, "The Generalised NMSSM at One Loop: Fine Tuning and Phenomenology," JHEP, vol. 1208, p. 074, 2012, 1205.1509.

[16] T. Gherghetta, B. von Harling, A. D. Medina, and M. A. Schmidt, "The Scale-Invariant NMSSM and the 126 GeV Higgs Boson," JHEP, vol. 1302, p. 032, 2013, 1212.5243.

[17] M. Perelstein and B. Shakya, "XENON100 implications for naturalness in the MSSM, NMSSM, and $\lambda$ supersymmetry model," Phys.Rev., vol. D88, no. 7, p. 075003, 2013, 1208.0833.

[18] D. Kim, P. Athron, C. Balázs, B. Farmer, and E. Hutchison, "Bayesian naturalness of the CMSSM and CNMSSM," Phys.Rev., vol. D90, no. 5, p. 055008, 2014, 1312.4150.

[19] A. Kaminska, G. G. Ross, K. Schmidt-Hoberg, and F. Staub, "A precision study of the fine tuning in the DiracNMSSM," JHEP, vol. 1406, p. 153, 2014, 1401.1816.

[20] M. Y. Binjonaid and S. F. King, "Naturalness of scale-invariant NMSSMs with and without extra matter," Phys.Rev., vol. D90, no. 5, p. 055020, 2014, 1403.2088.

[21] H. Dreiner, F. Staub, and A. Vicente, "General NMSSM signatures at the LHC," Phys.Rev., vol. D87, no. 3, p. 035009, 2013, 1211.6987.

[22] S. King, M. Mühlleitner, R. Nevzorov, and K. Walz, "Natural NMSSM Higgs Bosons," Nucl.Phys., vol. B870, pp. 323-352, 2013, 1211.5074 .

[23] S. King, M. Muhlleitner, R. Nevzorov, and K. Walz, "Discovery Prospects for NMSSM Higgs Bosons at the High-Energy Large Hadron Collider," Phys.Rev., vol. D90, no. 9, p. 095014, 2014, 1408.1120.

[24] U. Ellwanger and A. M. Teixeira, "NMSSM with a singlino LSP: possible challenges for searches for supersymmetry at the LHC," JHEP, vol. 1410, p. 113, 2014, 1406.7221.

[25] J. Espinosa and M. Quiros, "Two loop radiative corrections to the mass of the lightest Higgs boson in supersymmetric standard models," Phys.Lett., vol. B266, pp. 389-396, 1991.

[26] J. Espinosa and M. Quiros, "Upper bounds on the lightest Higgs boson mass in general supersymmetric Standard Models," Phys.Lett., vol. B302, pp. 51-58, 1993, hep-ph/9212305

[27] T. Elliott, S. King, and P. White, "Supersymmetric Higgs bosons at the limit," Phys.Lett., vol. B305, pp. 71-77, 1993, hep-ph/9302202.

[28] U. Ellwanger, "Radiative corrections to the neutral Higgs spectrum in supersymmetry with a gauge singlet," Phys.Lett., vol. B303, pp. 271-276, 1993, hep-ph/9302224

[29] P. Pandita, "Radiative corrections to the scalar Higgs masses in a nonminimal supersymmetric Standard Model," Z.Phys., vol. C59, pp. 575-584, 1993.

[30] T. Elliott, S. King, and P. White, "Squark contributions to Higgs boson masses in the next-to-minimal supersymmetric standard model," Phys.Lett., vol. B314, pp. 56-63, 1993, hep-ph/9305282.

[31] T. Elliott, S. King, and P. White, "Radiative corrections to Higgs boson masses in the next-to-minimal supersymmetric Standard Model," Phys.Rev., vol. D49, pp. 2435-2456, 1994, hep-ph/9308309

[32] G. Yeghian, "Upper bound on the lightest Higgs mass in supersymmetric theories," Acta Phys.Slov., vol. 49, p. 823, 199, hep-ph/9904488

[33] U. Ellwanger and C. Hugonie, "Masses and couplings of the lightest Higgs bosons in the (M+1) SSM," Eur.Phys.J., vol. C25, pp. 297-305, 2002, hep-ph/9909260

[34] U. Ellwanger and C. Hugonie, "Yukawa induced radiative corrections to the lightest Higgs boson mass in the NMSSM," Phys.Lett., vol. B623, pp. 93-103, 2005, hep-ph/0504269

[35] S. P. Martin, "Two loop effective potential for a general renormalizable theory and softly broken supersymmetry," Phys.Rev., vol. D65, p. 116003, 2002, hep-ph/0111209.

[36] G. Degrassi and P. Slavich, "On the radiative corrections to the neutral Higgs boson masses in the NMSSM," Nucl.Phys., vol. B825, pp. 119-150, 2010, 0907.4682.

[37] F. Staub, W. Porod, and B. Herrmann, "The Electroweak sector of the NMSSM at the one-loop level," JHEP, vol. 1010, p. 040, 2010, 1007.4049. 
[38] K. Ender, T. Graf, M. Muhlleitner, and H. Rzehak, "Analysis of the NMSSM Higgs Boson Masses at One-Loop Level," Phys.Rev., vol. D85, p. 075024, 2012, 1111.4952.

[39] T. Graf, R. Grober, M. Muhlleitner, H. Rzehak, and K. Walz, "Higgs Boson Masses in the Complex NMSSM at One-Loop Level," JHEP, vol. 1210, p. 122, 2012, 1206.6806.

[40] M. D. Goodsell, K. Nickel, and F. Staub, "Two-loop corrections to the Higgs masses in the NMSSM," Phys.Rev., vol. D91, no. 3, p. 035021, 2015, 1411.4665.

[41] M. Muhlleitner, D. T. Nhung, H. Rzehak, and K. Walz, "Two-Loop Contributions of the Order $\mathcal{O}\left(\alpha_{t} \alpha_{s}\right)$ to the Masses of the Higgs Bosons in the CP-Violating NMSSM," JHEP, vol. 1505, p. 128, 2015, 1412.0918.

[42] U. Ellwanger, J. F. Gunion, and C. Hugonie, "NMHDECAY: A Fortran code for the Higgs masses, couplings and decay widths in the NMSSM," JHEP, vol. 0502, p. 066, 2005, hep-ph/0406215.

[43] U. Ellwanger and C. Hugonie, "NMHDECAY 2.0: An Updated program for sparticle masses, Higgs masses, couplings and decay widths in the NMSSM," Comput.Phys.Commun., vol. 175, pp. 290-303, 2006, hep-ph/0508022.

[44] U. Ellwanger and C. Hugonie, "NMSPEC: A Fortran code for the sparticle and Higgs masses in the NMSSM with GUT scale boundary conditions," Comput.Phys.Commun., vol. 177, pp. 399-407, 2007, hep-ph/0612134

[45] B. Allanach, "SOFTSUSY: a program for calculating supersymmetric spectra," Comput.Phys.Commun., vol. 143, pp. 305-331, 2002, hep-ph/0104145

[46] B. Allanach, P. Athron, L. C. Tunstall, A. Voigt, and A. Williams, "Next-to-Minimal SOFTSUSY," Comput.Phys.Commun., vol. 185, pp. 2322-2339, 2014, 1311.7659.

[47] B. C. Allanach, A. Bednyakov, and R. Ruiz de Austri, "Higher order corrections and unification in the minimal supersymmetric standard model: SOFTSUSY3.5," Comput. Phys. Commun., vol. 189, pp. 192-206, 2015, 1407.6130.

[48] J. Baglio, R. Gröber, M. Mühlleitner, D. Nhung, H. Rzehak, et al., "NMSSMCALC: A Program Package for the Calculation of Loop-Corrected Higgs Boson Masses and Decay Widths in the (Complex) NMSSM," Comput.Phys.Commun., vol. 185, no. 12, pp. 3372-3391, 2014, 1312.4788.

[49] P. Athron, J.-h. Park, D. Stöckinger, and A. Voigt, "FlexibleSUSY - A spectrum generator generator for supersymmetric models," Comput.Phys.Commun., vol. 190, pp. 139-172, 2015, 1406.2319.

[50] W. Porod, "SPheno, a program for calculating supersymmetric spectra, SUSY particle decays and SUSY particle production at e+ e- colliders," Comput.Phys.Commun., vol. 153, pp. 275-315, 2003, hep-ph/0301101

[51] W. Porod and F. Staub, "SPheno 3.1: Extensions including flavour, CP-phases and models beyond the MSSM," Comput.Phys.Commun., vol. 183, pp. 2458-2469, 2012, 1104.1573.

[52] F. Staub, "SARAH," 2008, 0806.0538.

[53] F. Staub, "From Superpotential to Model Files for FeynArts and CalcHep/CompHep," Comput.Phys.Commun., vol. 181, pp. 1077-1086, 2010, 0909.2863.

[54] F. Staub, "Automatic Calculation of supersymmetric Renormalization Group Equations and Self Energies," Comput.Phys.Commun., vol. 182, pp. 808-833, 2011, 1002.0840.

[55] F. Staub, "SARAH 3.2: Dirac Gauginos, UFO output, and more," Computer Physics Communications, vol. 184, pp. pp. 1792-1809, 2013, 1207.0906.

[56] F. Staub, "SARAH 4: A tool for (not only SUSY) model builders," Comput.Phys.Commun., vol. 185, pp. 17731790, 2014, 1309.7223.

[57] B. Allanach, A. Djouadi, J. Kneur, W. Porod, and P. Slavich, "Precise determination of the neutral Higgs boson masses in the MSSM," JHEP, vol. 0409, p. 044, 2004, hep-ph/0406166

[58] B. Allanach, S. Kraml, and W. Porod, "Theoretical uncertainties in sparticle mass predictions from computational tools," JHEP, vol. 0303, p. 016, 2003, hep-ph/0302102

[59] J. E. Kim and H. P. Nilles, "The mu Problem and the Strong CP Problem," Phys.Lett., vol. B138, p. 150, 1984.

[60] S. Abel, S. Sarkar, and P. White, "On the cosmological domain wall problem for the minimally extended supersymmetric standard model," Nucl.Phys., vol. B454, pp. 663-684, 1995, hep-ph/9506359.

[61] P. Z. Skands, B. Allanach, H. Baer, C. Balazs, G. Belanger, et al., "SUSY Les Houches accord: Interfacing SUSY spectrum calculators, decay packages, and event generators," JHEP, vol. 0407, p. 036, 2004, hep-ph/0311123

[62] B. Allanach, C. Balazs, G. Belanger, M. Bernhardt, F. Boudjema, et al., "SUSY Les Houches Accord 2," Comput.Phys.Commun., vol. 180, pp. 8-25, 2009, 0801.0045.

[63] A. Djouadi, J. Kalinowski, and M. Spira, "HDECAY: A Program for Higgs boson decays in the standard model and its supersymmetric extension," Comput.Phys.Commun., vol. 108, pp. 56-74, 1998, hep-ph/9704448

[64] A. Djouadi, M. Muhlleitner, and M. Spira, "Decays of supersymmetric particles: The Program SUSY-HIT (SUspect-SdecaY-Hdecay-InTerface)," Acta Phys.Polon., vol. B38, pp. 635-644, 2007, hep-ph/0609292.

[65] J. M. Butterworth et al., "THE TOOLS AND MONTE CARLO WORKING GROUP Summary Report from the Les Houches 2009 Workshop on TeV Colliders," in Physics at TeV colliders. Proceedings, 6th Workshop, dedicated to Thomas Binoth, Les Houches, France, June 8-26, 2009, $2010,1003.1643$.

[66] F. Domingo, "A New Tool for the study of the CP-violating NMSSM," JHEP, vol. 06, p. 052, 2015, 1503.07087.

[67] A. Brignole, G. Degrassi, P. Slavich, and F. Zwirner, "On the O(alpha $\left.(\overline{\mathrm{t}})^{* *} 2\right)$ two loop corrections to the neutral Higgs boson masses in the MSSM," Nucl.Phys., vol. B631, pp. 195-218, 2002, hep-ph/0112177

[68] A. Dedes and P. Slavich, "Two loop corrections to radiative electroweak symmetry breaking in the MSSM," Nucl.Phys., vol. B657, pp. 333-354, 2003, hep-ph/0212132

[69] A. Dedes, G. Degrassi, and P. Slavich, "On the two loop Yukawa corrections to the MSSM Higgs boson masses at large tan beta," Nucl.Phys., vol. B672, pp. 144-162, 2003, hep-ph/0305127

[70] D. Das, U. Ellwanger, and A. M. Teixeira, "NMSDECAY: A Fortran Code for Supersymmetric Particle Decays in the Next-to-Minimal Supersymmetric Standard Model," Comput.Phys.Commun., vol. 183, pp. 774-779, 2012, 
1106.5633 .

[71] M. Muhlleitner, A. Djouadi, and Y. Mambrini, "SDECAY: A Fortran code for the decays of the supersymmetric particles in the MSSM," Comput.Phys.Commun., vol. 168, pp. 46-70, 2005, hep-ph/0311167

[72] F. Domingo and U. Ellwanger, "Updated Constraints from B Physics on the MSSM and the NMSSM," JHEP, vol. 0712, p. 090, 2007, 0710.3714

[73] F. Domingo and U. Ellwanger, "Constraints from the Muon g-2 on the Parameter Space of the NMSSM," JHEP, vol. 0807, p. 079, 2008, 0806.0733.

[74] S. Schael et al., "Search for neutral MSSM Higgs bosons at LEP," Eur.Phys.J., vol. C47, pp. 547-587, 2006, hep-ex/0602042

[75] G. Belanger, B. Dumont, U. Ellwanger, J. Gunion, and S. Kraml, "Global fit to Higgs signal strengths and couplings and implications for extended Higgs sectors," Phys.Rev., vol. D88, p. 075008, 2013, 1306.2941.

[76] A. Delgado, G. Giudice, and P. Slavich, "Dynamical mu term in gauge mediation," Phys.Lett., vol. B653, pp. 424433, 2007, 0706.3873.

[77] S. P. Martin and M. T. Vaughn, "Two loop renormalization group equations for soft supersymmetry breaking couplings," Phys.Rev., vol. D50, p. 2282, 1994, hep-ph/9311340

[78] Y. Yamada, "Two loop renormalization group equations for soft SUSY breaking scalar interactions: Supergraph method," Phys.Rev., vol. D50, pp. 3537-3545, 1994, hep-ph/9401241

[79] I. Jack and D. Jones, "Renormalization of the Fayet-Iliopoulos D term," Phys.Lett., vol. B473, pp. 102-108, 2000, hep-ph/9911491

[80] I. Jack, D. Jones, and S. Parsons, "The Fayet-Iliopoulos D term and its renormalization in softly broken supersymmetric theories," Phys.Rev., vol. D62, p. 125022, 2000, hep-ph/0007291.

[81] R. M. Fonseca, M. Malinsky, W. Porod, and F. Staub, "Running soft parameters in SUSY models with multiple U(1) gauge factors," Nucl.Phys., vol. B854, pp. 28-53, 2012, 1107.2670.

[82] M. D. Goodsell, "Two-loop RGEs with Dirac gaugino masses," JHEP, vol. 1301, p. 066, 2013, 1206.6697.

[83] M. Sperling, D. Stöckinger, and A. Voigt, "Renormalization of vacuum expectation values in spontaneously broken gauge theories," JHEP, vol. 1307, p. 132, 2013, 1305.1548.

[84] M. Sperling, D. Stockinger, and A. Voigt, "Renormalization of vacuum expectation values in spontaneously broken gauge theories: Two-loop results," JHEP, vol. 1401, p. 068, 2014, 1310.7629.

[85] M. D. Goodsell, K. Nickel, and F. Staub, "Two-Loop Higgs mass calculations in supersymmetric models beyond the MSSM with SARAH and SPheno," Eur. Phys. J., vol. C75, no. 1, p. 32, 2015, 1411.0675.

[86] M. Goodsell, K. Nickel, and F. Staub, "Generic two-loop Higgs mass calculation from a diagrammatic approach," Eur. Phys. J., vol. C75, no. 6, p. 290, 2015, 1503.03098.

[87] S. P. Martin, "Two loop scalar self energies in a general renormalizable theory at leading order in gauge couplings," Phys.Rev., vol. D70, p. 016005, 2004, hep-ph/0312092

[88] S. P. Martin, "Evaluation of two loop selfenergy basis integrals using differential equations," Phys.Rev., vol. D68, p. 075002,2003, hep-ph/0307101

[89] S. P. Martin, "Two-loop scalar self-energies and pole masses in a general renormalizable theory with massless gauge bosons," Phys.Rev., vol. D71, p. 116004, 2005, hep-ph/0502168

[90] W. Porod, F. Staub, and A. Vicente, "A Flavor Kit for BSM models," Eur. Phys. J., vol. C74, no. 8, p. 2992, 2014, 1405.1434 .

[91] J. R. Ellis, K. Enqvist, D. V. Nanopoulos, and F. Zwirner, "Observables in Low-Energy Superstring Models," Mod.Phys.Lett., vol. A1, p. 57, 1986.

[92] R. Barbieri and G. Giudice, "Upper Bounds on Supersymmetric Particle Masses," Nucl.Phys., vol. B306, p. 63, 1988.

[93] P. Bechtle, O. Brein, S. Heinemeyer, G. Weiglein, and K. E. Williams, "HiggsBounds: Confronting Arbitrary Higgs Sectors with Exclusion Bounds from LEP and the Tevatron," Comput.Phys.Commun., vol. 181, pp. 138-167, 2010, 0811.4169.

[94] P. Bechtle, O. Brein, S. Heinemeyer, G. Weiglein, and K. E. Williams, "HiggsBounds 2.0.0: Confronting Neutral and Charged Higgs Sector Predictions with Exclusion Bounds from LEP and the Tevatron," Comput.Phys.Commun., vol. 182, pp. 2605-2631, 2011, 1102.1898.

[95] P. Bechtle, O. Brein, S. Heinemeyer, O. Stål, T. Stefaniak, et al., "HiggsBounds - 4: Improved Tests of Extended Higgs Sectors against Exclusion Bounds from LEP, the Tevatron and the LHC," Eur.Phys.J., vol. C74, no. 3, p. 2693, 2014, 1311.0055

[96] P. Bechtle, S. Heinemeyer, O. Stål, T. Stefaniak, and G. Weiglein, "HiggsSignals: Confronting arbitrary Higgs sectors with measurements at the Tevatron and the LHC," Eur.Phys.J., vol. C74, no. 2, p. 2711, 2014, 1305.1933.

[97] J. Alwall, M. Herquet, F. Maltoni, O. Mattelaer, and T. Stelzer, "MadGraph 5 : Going Beyond," JHEP, vol. 1106 , p. $128,2011,1106.0522$.

[98] J. Alwall, R. Frederix, S. Frixione, V. Hirschi, F. Maltoni, et al., "The automated computation of tree-level and next-to-leading order differential cross sections, and their matching to parton shower simulations," JHEP, vol. 1407, p. 079, 2014, 1405.0301 .

[99] W. Kilian, T. Ohl, and J. Reuter, "WHIZARD: Simulating Multi-Particle Processes at LHC and ILC," Eur.Phys.J., vol. C71, p. 1742, 2011, 0708.4233.

[100] M. Moretti, T. Ohl, and J. Reuter, "O'Mega: An Optimizing matrix element generator," 2001, hep-ph/0102195

[101] F. Staub, T. Ohl, W. Porod, and C. Speckner, "A Tool Box for Implementing Supersymmetric Models," Comput.Phys.Commun., vol. 183, pp. 2165-2206, 2012, 1109.5147.

[102] V. D. Barger, M. Berger, and P. Ohmann, "The Supersymmetric particle spectrum," Phys.Rev., vol. D49, pp. 49084930, 1994, hep-ph/9311269 
[103] S. Heinemeyer, W. Hollik, and G. Weiglein, "FeynHiggs: A Program for the calculation of the masses of the neutral CP even Higgs bosons in the MSSM," Comput.Phys.Commun., vol. 124, pp. 76-89, 2000, hep-ph/9812320

[104] S. Heinemeyer, W. Hollik, and G. Weiglein, "The Masses of the neutral CP - even Higgs bosons in the MSSM: Accurate analysis at the two loop level," Eur.Phys.J., vol. C9, pp. 343-366, 1999, hep-ph/9812472

[105] G. Degrassi, S. Heinemeyer, W. Hollik, P. Slavich, and G. Weiglein, "Towards high precision predictions for the MSSM Higgs sector," Eur.Phys.J., vol. C28, pp. 133-143, 2003, hep-ph/0212020

[106] M. Frank, T. Hahn, S. Heinemeyer, W. Hollik, H. Rzehak, et al., "The Higgs Boson Masses and Mixings of the Complex MSSM in the Feynman-Diagrammatic Approach," JHEP, vol. 0702, p. 047, 2007, hep-ph/0611326.

[107] T. Hahn, S. Heinemeyer, W. Hollik, H. Rzehak, and G. Weiglein, "High-precision predictions for the light CP-even Higgs Boson Mass of the MSSM," Phys.Rev.Lett., vol. 112, p. 141801, 2014, 1312.4937.

[108] S. King, M. Muhlleitner, and R. Nevzorov, "NMSSM Higgs Benchmarks Near 125 GeV," Nucl.Phys., vol. B860, pp. 207-244, 2012, 1201.2671.

[109] L. J. Hall, D. Pinner, and J. T. Ruderman, "A Natural SUSY Higgs Near 126 GeV," JHEP, vol. 1204, p. 131, 2012, 1112.2703 .

[110] D. M. Pierce, J. A. Bagger, K. T. Matchev, and R.-j. Zhang, "Precision corrections in the minimal supersymmetric standard model," Nucl.Phys., vol. B491, pp. 3-67, 1997, hep-ph/9606211

[111] L. Avdeev and M. Y. Kalmykov, "Pole masses of quarks in dimensional reduction," Nucl.Phys., vol. B502, pp. 419435, 1997, hep-ph/9701308

[112] A. Bednyakov, A. Onishchenko, V. Velizhanin, and O. Veretin, "Two loop O(alpha-s**2) MSSM corrections to the pole masses of heavy quarks," Eur.Phys.J., vol. C29, pp. 87-101, 2003, hep-ph/0210258

[113] F. Staub, "Exploring new models in all detail with SARAH," Adv. High Energy Phys., vol. 2015, p. 840780, 2015, 1503.04200 .

[114] S. Fanchiotti, B. A. Kniehl, and A. Sirlin, "Incorporation of QCD effects in basic corrections of the electroweak theory," Phys.Rev., vol. D48, pp. 307-331, 1993, hep-ph/9212285

[115] G. Degrassi, S. Fanchiotti, and A. Sirlin, "Relations Between the On-shell and Ms Frameworks and the M $(W)$ M (Z) Interdependence," Nucl.Phys., vol. B351, pp. 49-69, 1991.

[116] J. Grifols and J. Sola, "One Loop Renormalization of the Electroweak Parameters in $N=1$ Supersymmetry," Nucl.Phys., vol. B253, p. 47, 1985.

[117] P. H. Chankowski, A. Dabelstein, W. Hollik, W. M. Mosle, S. Pokorski, et al., "Delta R in the MSSM," Nucl.Phys., vol. B417, pp. 101-129, 1994.

[118] C. Beskidt, W. de Boer, and D. Kazakov, "A comparison of the Higgs sectors of the CMSSM and NMSSM for a 126 GeV Higgs boson," Phys.Lett., vol. B726, pp. 758-766, 2013, 1308.1333.

[119] S. P. Martin, "Three-loop Standard Model effective potential at leading order in strong and top Yukawa couplings," Phys.Rev., vol. D89, p. 013003, 2014, 1310.7553.

[120] J. Elias-Miro, J. Espinosa, and T. Konstandin, "Taming Infrared Divergences in the Effective Potential," JHEP, vol. 1408 , p. 034, 2014, 1406.2652.

[121] S. P. Martin, "Taming the Goldstone contributions to the effective potential," Phys.Rev., vol. D90, p. 016013, 2014, 1406.2355.

[122] A. Djouadi, J.-L. Kneur, and G. Moultaka, "SuSpect: A Fortran code for the supersymmetric and Higgs particle spectrum in the MSSM," Comput.Phys.Commun., vol. 176, pp. 426-455, 2007, hep-ph/0211331

[123] P. Draper, G. Lee, and C. E. M. Wagner, "Precise estimates of the Higgs mass in heavy supersymmetry," Phys.Rev., vol. D89, no. 5, p. 055023, 2014, 1312.5743 .

[124] E. Bagnaschi, G. F. Giudice, P. Slavich, and A. Strumia, "Higgs Mass and Unnatural Supersymmetry," JHEP, vol. 09, p. 092, 2014, 1407.4081.

[125] J. P. Vega and G. Villadoro, "SusyHD: Higgs mass Determination in Supersymmetry," JHEP, vol. 07, p. 159, 2015, 1504.05200 . 